University of Nebraska - Lincoln

DigitalCommons@University of Nebraska - Lincoln

Papers in the Earth and Atmospheric Sciences

Earth and Atmospheric Sciences, Department of

$12-4-2012$

\title{
Spatial Filter Approach to Evaluating the Role of Convection on the Evolution of a Mesoscale Vortex
}

Glenn A. Creighton

Robert E. Hart

Philip Cunningham

Follow this and additional works at: https://digitalcommons.unl.edu/geosciencefacpub

Part of the Earth Sciences Commons

This Article is brought to you for free and open access by the Earth and Atmospheric Sciences, Department of at DigitalCommons@University of Nebraska - Lincoln. It has been accepted for inclusion in Papers in the Earth and Atmospheric Sciences by an authorized administrator of DigitalCommons@University of Nebraska - Lincoln. 


\title{
A Spatial Filter Approach to Evaluating the Role of Convection on the Evolution of a Mesoscale Vortex
}

\author{
GLENN A. CREIGHTON \\ Air Force Weather Agency, United States Air Force, Offutt Air Force Base, Nebraska \\ ROBERT E. HART \\ Department of Earth, Ocean, and Atmospheric Science, The Florida State University, Tallahassee, Florida \\ PHILIP CUNNINGHAM \\ PDL Solutions (Europe) Ltd, Hexham, United Kingdom
}

(Manuscript received 2 July 2012, in final form 4 December 2012)

\begin{abstract}
A new spatial filter is proposed that exploits a spectral gap in power between the convective scale and the system ("vortex") scale during tropical cyclone (TC) genesis simulations. Using this spatial separation, this study analyzes idealized three-dimensional numerical simulations of deep moist convection in the presence of a symmetric midlevel vortex to quantify and understand the energy cascade between the objectively defined convective scale and system scale during the early stages of tropical cyclogenesis. The simulations neglect surface momentum, heat, and moisture fluxes to focus on generation and enhancement of vorticity within the interior to more completely close off the energy budget and to be consistent for comparison with prior benchmark studies of modeled TC genesis.

The primary contribution to system-scale intensification comes from the convergence of convective-scale vorticity that is supplied by vortical hot towers (VHTs). They contribute more than the convergence of system-scale vorticity to the spinup of vorticity in these simulations by an order of magnitude. Analysis of the change of circulation with time shows an initial strengthening of the surface vortex, closely followed by a growth of the mid- to upper-level circulation. This evolution precludes any possibility of a stratiform precipitation-induced top-down mechanism as the primary contributor to system-scale spinup in this simulation. Instead, an upscale cascade of rotational kinetic energy during vortex mergers is responsible for spinup of the simulated mesoscale vortex. The spatial filter employed herein offers an alternative approach to the traditional symmetry-asymmetry paradigm, acknowledges the highly asymmetric evolution of the systemscale vortex itself, and may prove useful to future studies on TC genesis.
\end{abstract}

\section{Introduction}

The problem of tropical cyclogenesis has received considerable attention over the past several decades, with increasing refinement of theories concerning the process. Despite the diversity of the approaches to tackling the problem, one aspect has largely remained constant among them: the choice of method for separating the vortex scale from the convective scale. In the vast majority of these studies, the "vortex" is typically

Corresponding author address: Glenn Creighton, HQ AFWA, 2WXG, 16WS/WXN, 101 Nelson Drive, Offutt Air Force Base, NE 68113-1023.

E-mail: glenn.creighton@offutt.af.mil separated from the "convective" scale by employing a filter that separates the symmetric flow from the asymmetric flow, respectively. While this approach has become standard and is relatively easily implemented, it is not clear whether it is the most physically sound choice, as discussed next.

\section{a. Separation of vortex scale from convective scale}

The earliest observational (Gray 1968, 1981), analytical (Adem 1956), and modeling studies (Anthes 1972) of tropical cyclones (TCs) have shown clearly that there are natural large-scale asymmetries to the TC itself. Wavenumber-1 gyres form in response to the variation of Coriolis across the vortex and are considered part of 
the vortex itself (Fiorino and Elsberry 1989). Horizontal and vertical shear can force wavenumber-1, -2 , and higher-order structure as part of the storm (Elsberry 1995). There are often spiral bands (Moon and Nolan 2010; Li and Wang 2012) that traverse less than half the storm azimuthally, vortex Rossby waves that exist on the strong vorticity gradient around the storm core (Montgomery and Kallenbach 1997), and the core itself can be highly asymmetric (Schubert et al. 1999). All of these structures contain significant asymmetric structure, and thus when conventional separation approaches are used, are partially or totally removed from the vortex field and incorrectly distributed into the "nonvortex" field.

During earlier stages of TC development, the symmetric component of the flow is obviously not as well defined as it is during the mature TC stage. There can be as much energy in the asymmetric component of the flow as there is in the symmetric component of the flow during these early stages, with the asymmetric-forced heating often playing a critical role in development (Simpson et al.1997; Nolan et al. 2007). Thus, for most of the life cycle of a TC, a symmetric-asymmetric scaleseparation approach does not achieve the goal of quantifying the evolution of the vortex independent of the remainder of the flow. Nonetheless, it is worth noting the obvious point here that there is no way to perfectly separate a vortex from its "environment" as such a separation does not exist physically, in particular at upper levels where outflow and outflow jets may not be bounded even if the cyclonic circulation is. This important caveat notwithstanding, the goal is to choose a vortex-convective scale-separation method that captures as much of the known broad-scale symmetric and asymmetric TC structural signatures.

Accordingly a scale-separation method is presented here that does not assume the TC vortex is symmetric and thus more completely captures the evolution. The separation proposed is based on a clear spectral gap in the power spectrum of modeled developing TCs. At wavelengths smaller than the spectral gap, the composite flow is called the convective scale; at wavelengths larger than the spectral gap, the composite flow is called the "system" or vortex scale. Further, by quantifying the energy as a function of wavelength (rather than just two mutually exclusive bins of symmetric and asymmetric) the energy cascades across the spectrum as well as across the gap itself can be quantified-something that was not possible with the conventional scale-separation approach.

\section{b. Application to tropical cyclogenesis}

With recent field experiments including the National Aeronautics and Space Administration (NASA)
African Monsoon Multidisciplinary Analyses (NAMMA), NASA Fourth Convection and Moisture Experiment (CAMEX-4), NASA Genesis and Rapid Intensification Project (GRIP), and the Pre-Depression Investigation of Cloud-Systems in the Tropics (PREDICT), there has been an aggressive attempt to evaluate TC genesis theories using both observations and numerical modeling. One of the recent theories for TC genesis was proposed by Bister and Emanuel (1997, hereafter BE97). In their case study on the genesis of Hurricane Guillermo (1991) they proposed a "top-down showerhead" mechanism. They suggested that midlevel cooling caused by large areas of stratiform precipitation initially creates a moist midlevel cold-core vortex and a subsidence-driven warmer, drier low-level anticyclone. As a result of surface divergence, this surface anticyclone is dispersed outward and the midlevel cyclone slowly descends to take its place. The now-cool, moist boundary layer creates a convectively unstable environment over the warm sea surface that triggers downdraft-free convection. This leads to the development and intensification of a warm-core cyclonic surface vortex. Nolan (2007) has since shown that the vortex squashing resulting from this subsidence prevents the midlevel vortex from descending as far as needed to focus low-level convection.

Montgomery et al. (2006, hereafter M06) proposed a "bottom up" theory for TC genesis in which vortical hot towers (VHTs; Hendricks et al. 2004) within an initially weak cold-core mesoscale convective vortex (MCV) provide a systemwide source of cyclonic vorticity and diabatic heating. This diabatic heating creates a quasi-balanced secondary circulation that aligns both system and VHT vorticity and is able to accelerate spinup of near-surface tangential winds. M06 incorporates three-dimensional convective events and accounts for upscale vorticity growth via multiple VHT vortex mergers. Later, Montgomery et al. (2009) proposed that this VHT pathway to TC genesis can also be extended to explain mature TC intensification. They argue that, although surface latent heat fluxes are essential for TC intensification, the wind-induced surface heat exchange (WISHE; Emanuel 1986; Rotunno and Emanuel 1987) mechanism itself is subdominant to the nearsurface convergence created by locally buoyant VHTs within the boundary layer.

While the above theories are not the only ones proposed for TC genesis (e.g., Ritchie and Holland 1997; Dunkerton et al. 2009), the research presented here is primarily motivated by the work of M06, its contrast to BE97, and the need to quantify that contrast in the context of an improved scale-separation method. In M06, reference is made to the notion of an upscale cascade of rotational kinetic energy from a wide distribution of 
VHTs to the system-scale vortex. M06 argued that the net effect of these VHTs was to "vortically prime the mesoscale environment, making the spinup into a tropical depression vortex more likely." They diagnosed that the primary reason for the system-scale increase in tangential momentum was a net radial influx of systemscale vorticity, or effectively that the spinup was caused by large-scale convergence of cyclonic vorticity. It is important to note that the "system scale" there was still defined by the purely symmetric flow and thus ignored considerable asymmetries that were incorrectly being assigned to the nonvortex field-perhaps influencing the cascade.

\section{c. Approach and goals of this study}

Accordingly, using the new scale-separation approach described in detail later, vorticity budget and energy cascade of a simulated vortex similar to M06 are analyzed. A symmetric midlevel cyclone of similar dimensions to those used by M06 is placed within a conditionally unstable nonsheared environment, is brought into gradient wind balance through integration, and is finally locally perturbed before evolving over a 48 -h period. To focus on the role of internal generation and destruction of vorticity, both surface friction and surface fluxes are set to zero in these numerical simulations; future studies will examine the impact of this surface modification on the results of the simulations. The intentional absence of surface friction and fluxes in these experiments guarantees that there will not be any intensification due to these processes that might detract from that goal. The reader is directed to experiments D1 and D2 in M06 as the nearest analogs to the simulations undertaken herein. Additionally, as this study focuses only on pre-WISHE vorticity development, it does not concentrate on explaining further TC intensification, but merely on quantifying the role of convective-scale processes on the enhancement of the embryonic systemscale vortex preceding TC status.

It is not the goal of this study to identify new theories for tropical cyclogenesis, but instead to reexamine M06like simulations in the context of a more physically appropriate spatial filter. The choice of filter permits the examination of the vorticity budget evolution for each of the two regimes identified by the filter and to examine the energy cascade across the gap between those regimes. These examinations allow for a more robust quantification of the results found and arguments made in M06 without the caveat of assuming a symmetric vortex. Additionally, evaluation of these results on a welltested set of prior simulations acts as an evaluation of the new filter technique proposed here and may serve as a new framework in which future TC modeling studies may be conducted.
This paper is organized as follows: Section 2 summarizes the experimental design and numerical model. Section 3 provides a description of the analysis techniques employed including a detailed description of the spatial filter. Section 4 presents the results of the control experiment and reviews the sensitivity experiments. Finally, section 5 summarizes these findings and discusses the possible implications.

\section{Experimental design and model description}

\section{a. Model summary}

The simulations described in this paper are run with the Weather Research and Forecasting (WRF), version 1.2 (Skamarock et al. 2001), numerical weather prediction model. This version of WRF employs a Cartesian vertical coordinate system that readily conforms to the vortex initialization techniques used here, whereas later versions employ a mass coordinate. The domain measures $600 \mathrm{~km} \times 600 \mathrm{~km} \times 24 \mathrm{~km}$ and has a 2-km $(0.4 \mathrm{~km})$ horizontal (vertical) grid spacing. ${ }^{1}$ Open lateral boundary conditions allow gravity waves to leave the domain, while winds adjacent to the lateral boundaries dictate inflow. Rayleigh damping removes vertically propagating gravity waves in the upper $6 \mathrm{~km}$ of the domain. ${ }^{2}$ All simulations have a free-slip lower boundary condition.

This study makes use of the following physical parameterizations: Lin et al. (1983) for cloud microphysics, a thermal diffusion scheme for temperature, and a 1.5order turbulent kinetic energy closure scheme for subgridscale turbulence. Convection evolves within the model without the assistance of surface, boundary layer, or convective parameterizations. These simulations do not include radiation, keeping them as idealized as possible, consistent with D1 and D2 from M06, and ensuring a thermodynamically closed system.

\section{b. Experimental design}

The control experiment (A1; Table 1) simulates a moist vortex with a radius of maximum winds (RMW) of $75 \mathrm{~km}$ and maximum tangential velocity of $8 \mathrm{~m} \mathrm{~s}^{-1}$, following the specifications in M06. A localized 2-K warm temperature perturbation initiates convection $50 \mathrm{~km}$ to the west of the vortex center. The modeled vortex

\footnotetext{
${ }^{1}$ Higher-resolution sensitivity experiments confirm that this grid spacing adequately captures the storm-scale phenomena of interest. For more information on the effect of grid spacing on developing convection in a cloud resolving model, refer to Petch (2006).

${ }^{2}$ The Rayleigh damping coefficient used for these experiments is $0.2 \mathrm{~s}^{-1}$
} 
TABLE 1. WRF vortex experiments-initial conditions. The term $N_{p}$ is the number of initial temperature perturbations.

\begin{tabular}{|c|c|c|c|c|}
\hline No. & Name & Enhanced $q_{v}$ & $N_{p}$ & Notes \\
\hline A1 & Control & No & 1 & $\begin{array}{l}V_{m}=8 \mathrm{~m} \mathrm{~s}^{-1} \text { at } 3.5 \mathrm{~km}, r_{m}=75 \mathrm{~km} . \text { Near-surface mean tangential wind is } 4 \mathrm{~m} \mathrm{~s}^{-1} \\
\text { at } 24 \mathrm{~h} \text { and } 6 \mathrm{~m} \mathrm{~s}^{-1} \text { at } 48 \mathrm{~h} .\end{array}$ \\
\hline $\mathrm{A} 2$ & Moist & Yes & 1 & $\begin{array}{l}\text { As in control, except with enhanced } q_{v} \text { at vortex center. Near-surface mean tangential } \\
\text { wind is } 10 \mathrm{~m} \mathrm{~s}^{-1} \text { at } 24 \mathrm{~h} \text { and } 8 \mathrm{~m} \mathrm{~s}^{-1} \text { at } 48 \mathrm{~h} \text {. }\end{array}$ \\
\hline B1 & Random & No & 4 & $\begin{array}{l}\text { As in control, except with multiple perturbations randomly placed within the vortex. } \\
\text { Near-surface mean tangential wind is } 6 \mathrm{~m} \mathrm{~s}^{-1} \text { at } 24 \mathrm{~h} \text { and } 7 \mathrm{~m} \mathrm{~s}^{-1} \text { at } 48 \mathrm{~h} \text {. }\end{array}$ \\
\hline B2 & Moist random & Yes & 4 & $\begin{array}{l}\text { As in B1, except with enhanced } q_{v} \text { at vortex center. Near-surface mean tangential } \\
\text { wind is } 8 \mathrm{~m} \mathrm{~s}^{-1} \text { at } 24 \mathrm{~h} \text { and } 14 \mathrm{~m} \mathrm{~s}^{-1} \text { at } 48 \mathrm{~h} \text {. }\end{array}$ \\
\hline
\end{tabular}

evolves over $48 \mathrm{~h}$, with no friction, heat, or moisture fluxes allowed from the surface.

Three sensitivity experiments complement and assess the robustness of the control experiment. The first of these (A2; Table 1) adds a moisture perturbation to the vortex at low to midlevels during initialization. The perturbation locally enhances convective available potential energy (CAPE) by approximately $300 \mathrm{~J} \mathrm{~kg}^{-1}$. The second sensitivity experiment (B1; Table 1 ) tests the system's sensitivity to the number of initial temperature perturbations within the initial vortex. A random number generator initializes the locations within the RMW of four distinct temperature perturbations, each having the same magnitude and structure as the control perturbation. The last experiment (B2; Table 1) is simply a combination of the previous two experiments.

\section{c. Initialization of the $M C V$}

As in M06, the Jordan (1958) mean Atlantic hurricane season sounding defines the ambient temperature and moisture profiles (Fig. 1). An MCV initialized in a manner identical to that in Trier et al. (2000) superimposes the background temperature profile and has the tangential flow given by

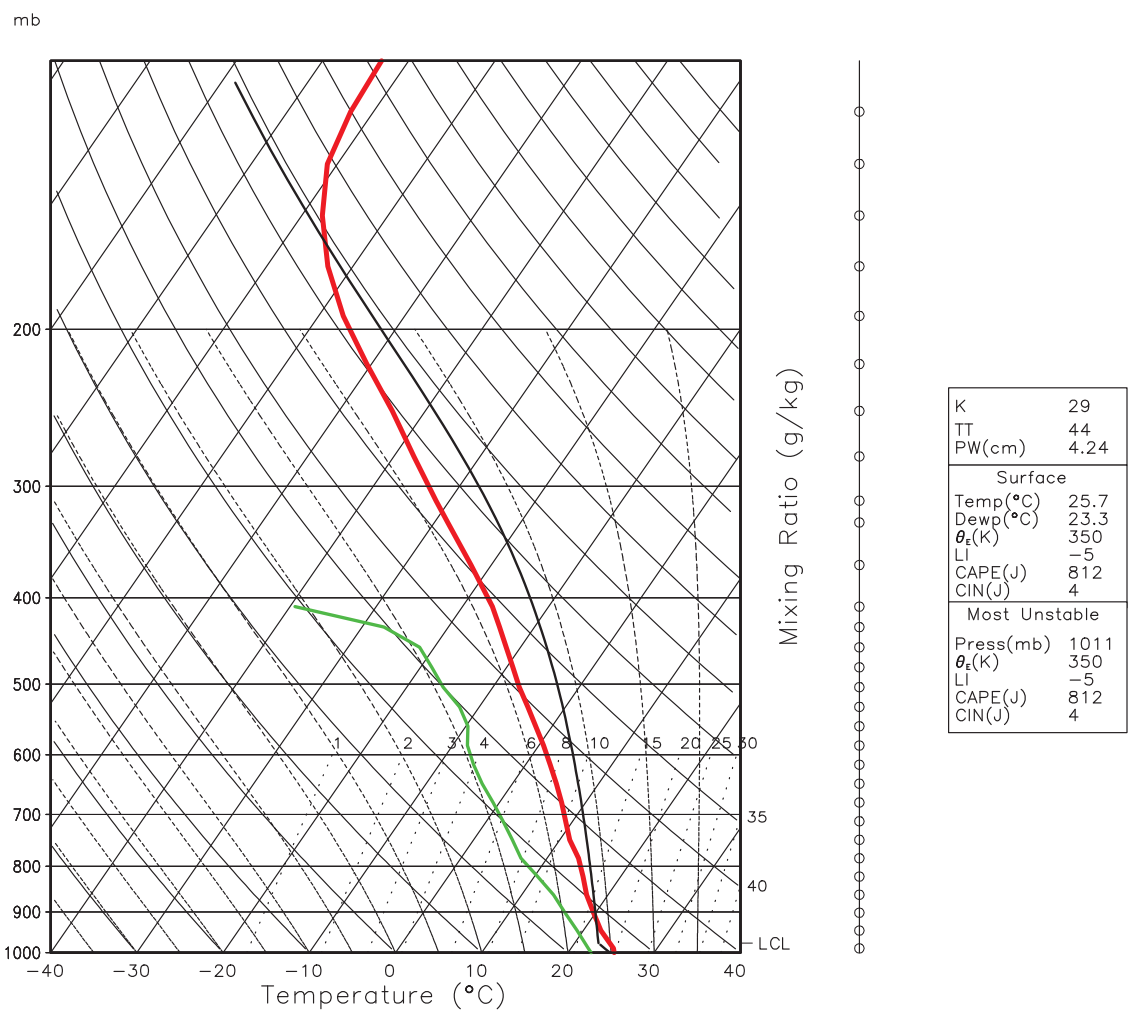

FIG. 1. Skew $T-\log p$ sounding of ambient temperature and moisture used in all experiments. The temperature sounding is the thick red line. The dewpoint sounding is the thick green line, and the thin solid black line denotes the lifted parcel ascent path. CAPE and other diagnostics are indicated in the key. 


$$
V(r, z)= \begin{cases}b(z) V_{m}\left(\frac{r}{r_{m}}\right), & r \leq r_{m} \\ b(z) V_{m}\left(\frac{r_{m}}{r}\right)^{c}, & r \geq r_{m}\end{cases}
$$

where $r$ is the distance from the center of the vortex and $V_{m}$ is the maximum tangential wind at the radius of maximum winds $r_{m}$. The Coriolis parameter $f$ is $7.292 \times$ $10^{-5} \mathrm{~s}^{-1}$ for all experiments, and $c=1+f r_{m} / V_{m}$. As in the control experiment within M06, $V_{m}=8 \mathrm{~m} \mathrm{~s}^{-1}$ and $r_{m}=75 \mathrm{~km}$ for the initial vortex (see Fig. 2a). The vertical vortex structure $b(z)$ is defined as

$$
b(z)=\exp \left[\frac{-\left(z-z_{m}\right)^{2}}{2 H^{2}}\right]
$$

where $z_{m}=3.5 \mathrm{~km}$ is the height of $V_{m}$ and $H=2 \mathrm{~km}$ is the Gaussian half-depth, the latter of which determines the vertical decay of the vortex from its center (Trier et al. 2000).

The hydrostatic equation also yields the perturbation potential temperature (Fig. 2b):

$$
\theta^{\prime}(x, y, z)=\frac{c_{p} \theta_{a}(z)^{2}}{g} \frac{\partial \pi^{\prime}}{\partial z}
$$

where $c_{p}$ is the specific heat capacity of dry air at constant pressure, $\theta_{a}(z)$ is the ambient potential temperature profile, $g$ is standard gravity, and the perturbation Exner function $\pi^{\prime}$ is obtained through the radial integration of gradient wind balance:

$$
\pi^{\prime}(r, z)= \begin{cases}\frac{-V_{m} b(z)}{c_{p} \theta_{a}(z)}\left[\frac{V_{m} b(z)}{2}\left(1+\frac{1}{c}-\frac{r^{2}}{r_{m}}\right)+f\left(\frac{r_{m}}{c-1}+\frac{r_{m}}{2}-\frac{r^{2}}{2 r_{m}}\right)\right], & r \leq r_{m} \\ \frac{-V_{m} b(z)}{c_{p} \theta_{a}(z)}\left[\frac{V_{m} b(z)}{2 c}\left(\frac{r_{m}}{r}\right)^{2 c}+\frac{f r_{m}^{c} r^{1-c}}{c-1}\right], & r \geq r_{m} .\end{cases}
$$

The addition of one or more localized heat sources stimulates initial convection within the vortex, with those perturbations $\theta_{\text {bub }}^{\prime}$ defined by a cosine function of the form

$$
\begin{aligned}
\theta_{\mathrm{bub}}^{\prime}(r, z)= & T_{\mathrm{bub}} \cos \left\{\frac{\pi}{2}\left[\left(\frac{r}{r_{\text {bub }}}\right)^{2}+\left(\frac{z-h_{\text {bub }}}{z_{\text {bub }}}\right)^{2}\right]^{1 / 2}\right\}^{2}, \\
& {\left[\left(\frac{r}{r_{\text {bub }}}\right)^{2}+\left(\frac{z-h_{\text {bub }}}{z_{\text {bub }}}\right)^{2}\right]^{1 / 2} \leq 1, }
\end{aligned}
$$

where $T_{\text {bub }}$ is the maximum temperature perturbation, set to $2 \mathrm{~K}$ for this study; $r_{\text {bub }}$ is the horizontal perturbation radius, set to $8 \mathrm{~km}$; $z_{\text {bub }}$ is the perturbation height, set to $2 \mathrm{~km}$; and $h_{\text {bub }}$ is the perturbation's vertical position, set to zero for a surface perturbation. The addition of a random noise of small amplitude $( \pm 5 \%$ of $\left.\theta_{\text {bub }}^{\prime}\right)$ to the initial temperature perturbations breaks any resonances caused by symmetry (Craig and Dörnbrack 2008).
For some experiments, following M06, a moisture perturbation $q_{v}^{\prime}(r, z)$ superimposes the ambient moisture profile, $q_{v, a}(z)$, such that

$$
\begin{aligned}
q_{v}^{\prime}(r, z)= & q_{\max } \cos \left\{\frac{\pi}{2}\left[\left(\frac{r}{r_{\max }}\right)^{2}+\left(\frac{z}{z_{\max }}\right)^{2}\right]^{1 / 2}\right\}, \\
& {\left[\left(\frac{r}{r_{\max }}\right)^{2}+\left(\frac{z}{z_{\max }}\right)^{2}\right]^{1 / 2} \leq 1 . }
\end{aligned}
$$

Here, the terms are analogous to (5), where $q_{\max }=$ $1.4 \mathrm{~g} \mathrm{~kg}^{-1}, r_{\max }=100 \mathrm{~km}$, and $z_{\max }=6 \mathrm{~km}$ (see Fig. $2 \mathrm{~d}$ ). An iterative technique employed over the domain hydrostatically rebalances pressure for moisture until a critical pressure perturbation tolerance of $1 \times 10^{-4} \mathrm{hPa}$ is achieved.

\section{Analysis techniques}

\section{a. Vorticity budgeting}

Examining the vorticity tendency equation enables diagnosis of the primary mechanisms responsible for vorticity generation and enhancement. The vorticity tendency equation in Cartesian coordinates is

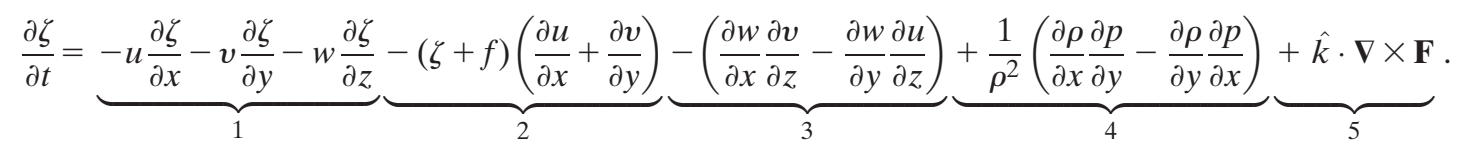


Here, term 1 is advection, term 2 is the divergence term, and term 3 is the tilting term. Terms 4 and 5 are the solenoidal and friction terms, respectively. As shown in M06, scale analysis indicates that at these scales, terms 4 and 5 are more than an order of magnitude smaller than terms 2 and 3.

This study concentrates on the divergence and tilting terms because its focus is primarily on vorticity generation and enhancement, not transport. Thus, the vorticity tendency due to each becomes

$$
\left.\frac{\partial \zeta}{\partial t}\right|_{\mathrm{DIV}}=-(\zeta+f)\left(\frac{\partial u}{\partial x}+\frac{\partial v}{\partial y}\right)
$$

$$
\left.\frac{\partial \zeta}{\partial t}\right|_{\mathrm{TT}}=-\left(\frac{\partial w}{\partial x} \frac{\partial v}{\partial z}-\frac{\partial w}{\partial y} \frac{\partial u}{\partial z}\right)
$$

To determine the relative contributions to (7) from the convective and system spatial scales, each state variable $F_{s}$ separates into spatially filtered means and perturbations from those means, such that

$$
F_{s}(x, y, z, t)=\overline{F_{s}}(x, y, z, t)+F_{s}^{\prime}(x, y, z, t),
$$

where overbars represent spatial averages and primes denote spatial perturbations from those averages. Applying (10) to (8) and (9) and taking the spatial mean yields the spatially filtered vorticity tendencies due to divergence,

$$
\left.\overline{\frac{\partial \zeta}{\partial t}}\right|_{\text {DIV }}=\underbrace{-\overline{(\bar{\zeta}+f)\left(\frac{\partial \bar{u}}{\partial x}+\frac{\partial \bar{v}}{\partial y}\right)}}_{\text {MMD }} \underbrace{-\overline{(\bar{\zeta}+f)\left(\frac{\partial u^{\prime}}{\partial x}+\frac{\partial v^{\prime}}{\partial y}\right)}}_{\text {MED }} \underbrace{-\overline{\zeta^{\prime}\left(\frac{\partial \bar{u}}{\partial x}+\frac{\partial \bar{v}}{\partial y}\right)}}_{\text {EMD }} \underbrace{-\overline{\zeta^{\prime}\left(\frac{\partial u^{\prime}}{\partial x}+\frac{\partial v^{\prime}}{\partial y}\right)}}_{\text {EED }},
$$

and tilting,

$$
\left.\overline{\frac{\partial \zeta}{\partial t}}\right|_{\text {TT }}=\underbrace{-\overline{\left(\frac{\partial \bar{w}}{\partial x} \frac{\partial \bar{v}}{\partial z}-\frac{\partial \bar{w}}{\partial y} \frac{\partial \bar{u}}{\partial z}\right)}}_{\text {MMT }}-\underbrace{-\overline{\left(\frac{\partial \bar{w}}{\partial x} \frac{\partial v^{\prime}}{\partial z}-\frac{\partial \bar{w}}{\partial y} \frac{\partial u^{\prime}}{\partial z}\right)}}_{\text {MET }}-\underbrace{\overline{\left(\frac{\partial w^{\prime}}{\partial x} \frac{\partial \bar{v}}{\partial z}-\frac{\partial w^{\prime}}{\partial y} \frac{\partial \bar{u}}{\partial z}\right)}}_{\text {EMT }}-\underbrace{-\overline{\left(\frac{\partial w^{\prime}}{\partial x} \frac{\partial v^{\prime}}{\partial z}-\frac{\partial w^{\prime}}{\partial y} \frac{\partial u^{\prime}}{\partial z}\right)}}_{\text {EET }},
$$

where EED is the eddy-eddy-divergence term (the eddy stretching of eddy vorticity) and EET is the eddy-eddytilting term (eddy tilting of eddy horizontal vorticity). MMD is the mean-mean-divergence term (the mean stretching of mean vorticity), and MMT is the meanmean-tilting term (the mean tilting of mean horizontal vorticity).

\section{b. Spatial filtering}

As mentioned earlier, a spatial filtering approach not common to this type of study is proposed here-seeking a more physical separation of vortex from convective scale. Specifically, a spatial filter calculates the mean terms in (10) using a two-dimensional weighted moving average. A window function-one that is zero valued outside of some set interval-defines the weight of this average. In this study, the filter separates low-wavenumber signals (i.e., spatial means) from the higher-order structures. The filter function is

$$
\bar{F}(x, y, z, t)=\frac{\sum_{j=-N / 2}^{N / 2} \sum_{i=-N / 2}^{N / 2} w_{i j} F\left(x+x_{i}, y+y_{j}, z, t\right)}{\sum_{j=-N / 2}^{N / 2} \sum_{i=-N / 2}^{N / 2} w_{i j}},
$$

where $F$ is the variable to be filtered, $w_{i j}$ is the window function, and $N$ is the width of the window function in grid points, referred to as the filter length. ${ }^{3}$ A cosine curve describes the window function:

$$
w_{i j}= \begin{cases}\cos \left(\frac{\pi \sqrt{i^{2}+j^{2}}}{N}\right), & \sqrt{i^{2}+j^{2}} \leq N \\ 0, & \sqrt{i^{2}+j^{2}}>N .\end{cases}
$$

Thus, points closer to the center have more weight, and those farther away have less weight. Sensitivity experiments (not shown) indicate that the shape of the window function is less important than choosing an appropriate filter length.

To separate the convective-scale signal from the systemscale signal, a cutoff wavenumber $K_{0}$ is chosen within the spectral gap between these two scales. Once the cutoff wavenumber is located, $N$ is set such that wavenumbers above the cutoff are attenuated and those below are preserved. Using a two-dimensional fast Fourier transform (FFT), for each experiment a representative sample from

\footnotetext{
${ }^{3}$ Note that $i$ and $j$ are centered about the point of interest requiring that $N$ be an integer multiple of 2 .
} 
(a)

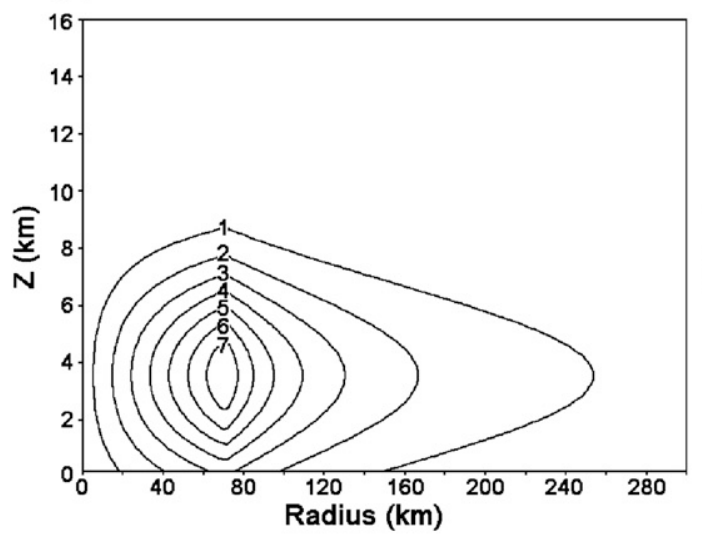

(c)

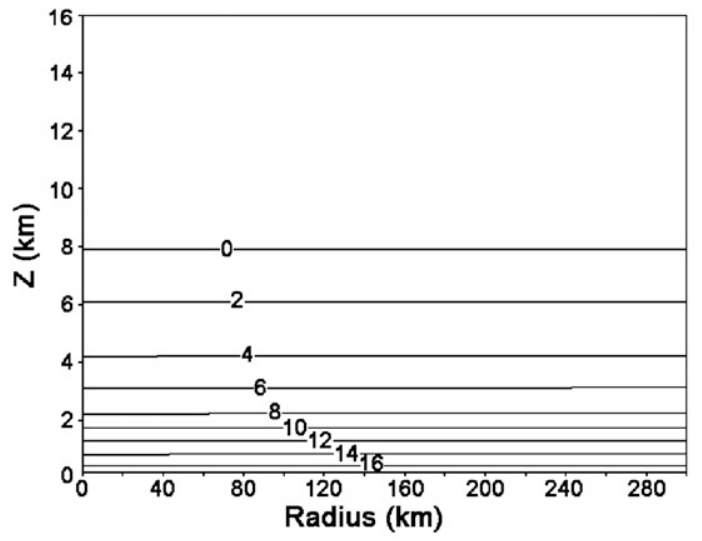

(e)

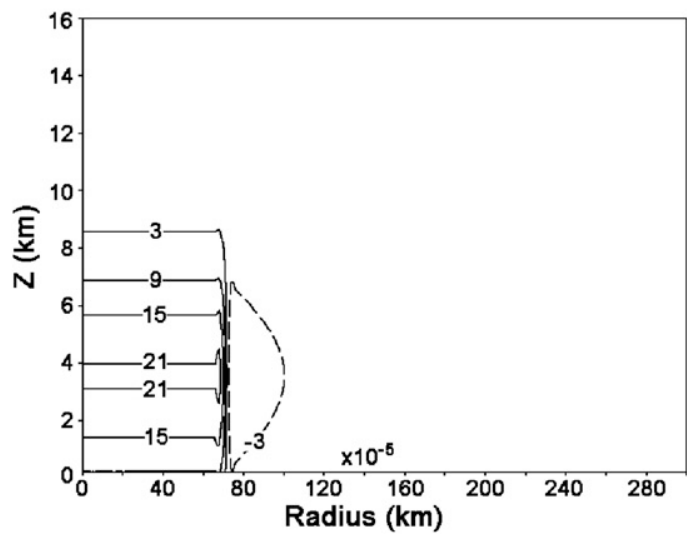

(b)

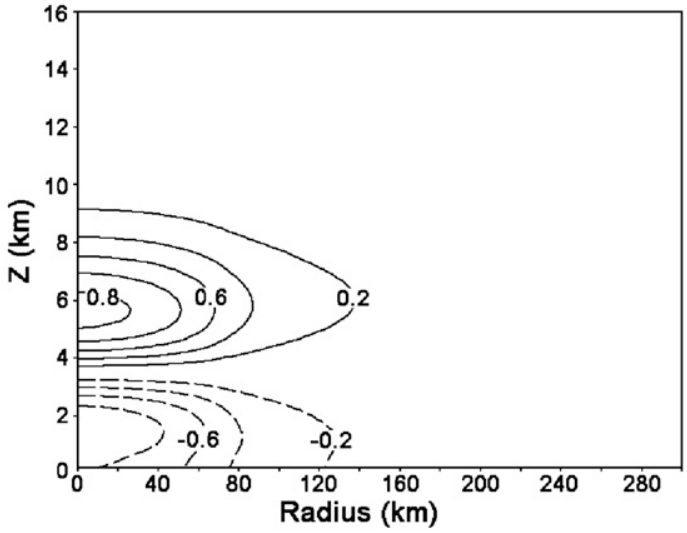

(d)

$q_{v}^{\prime}$

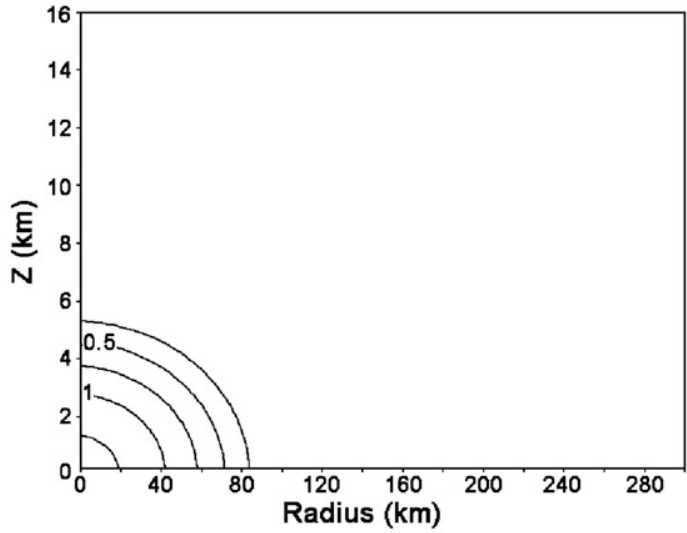

(f)

PV

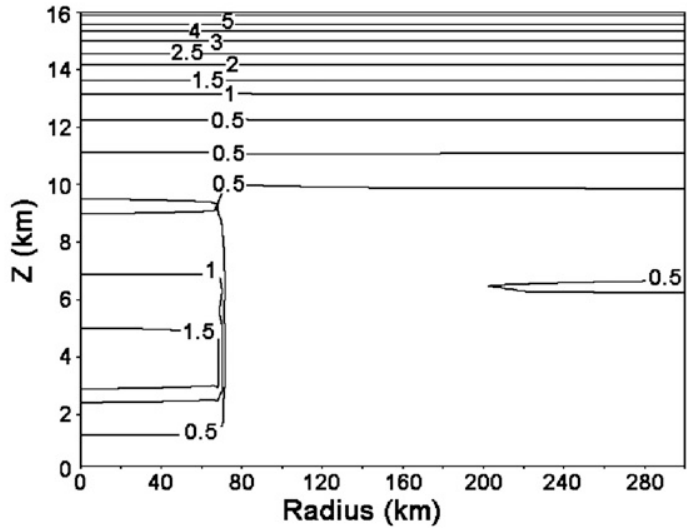

FIG. 2. Initial conditions: (a) tangential winds $\left(\mathrm{m} \mathrm{s}^{-1}\right.$ ), (b) perturbation potential temperature (K), (c) water vapor mixing ratio $\left(\mathrm{g} \mathrm{kg}^{-1}\right)$, (d) perturbation water vapor mixing ratio $\left(\mathrm{g} \mathrm{kg}^{-1}\right)$ (used in experiments $\mathrm{A} 2$ and $\mathrm{B} 2$ ), (e) relative vorticity $\left(\mathrm{s}^{-1}\right)$, and (f) potential vorticity (PV units).

the near-surface vorticity field is analyzed at each time and averaged to create a composite power spectrum. A representative power spectrum from the control experiment A1 (Fig. 3) shows a spectral gap that ranges from wavenumbers 5 to 20 (wavelengths $\lambda$ of $30-120 \mathrm{~km}$ ). As the initial vortex has a diameter of $150 \mathrm{~km}$ and convective updrafts are generally found from inspection to be less than $20 \mathrm{~km}$ wide, this gap is physically reasonable.

To minimize the impulse response of the filter, $K_{0}$ is chosen to be as large as possible within the spectral gap. Allowing for a little error in estimating the gap and slight variability between experiments, $K_{0}=15(\lambda=40 \mathrm{~km})$ is 


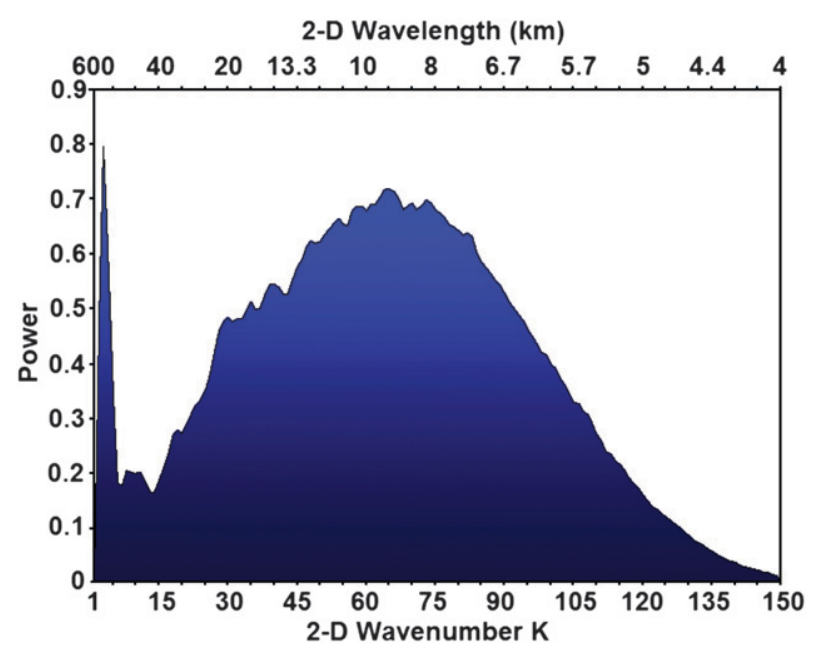

FIG. 3. Time-averaged normalized power spectrum of nearsurface $(200 \mathrm{~m})$ vorticity for experiment A1. Note the spectral gap between wavenumbers 5 and 20 .

chosen as the cutoff wavenumber in this study. Following guidance put forth by James (2010) for a similar filter, the filter length here was found through experimentation to be

$$
N \approx 1.25 \frac{K_{\max }}{K_{0}},
$$

where $K_{\max }$ is the domain-maximum wavenumber. Here, the filter length is found to be 12.5 (or $N=12$ to the nearest integer multiple of 2).

To determine the effectiveness of the filter in attenuating the high-wavenumber signal, the filter response is evaluated by running the FFT again on the filtered output and dividing by the unfiltered field. The blue line in Fig. 4 shows the filter response of the control run for $N=12$. The cutoff wavenumber is that at which the filter response intersects $-3 \mathrm{~dB}$, denoted by the thin dotted line. The thick dotted line shows an example of an ideal filter response at $K_{0}=15$. The $N=12$ filter effectively attenuates wavenumbers greater than $K_{0}=15$ but suffers from aliasing, apparent in the scalloping pattern outside the main lobe. Filtering a second time removes most visible signs of aliasing, but also decreases the cutoff frequency. This can be seen by comparing the two-pass $N=12$ (green) with the one-pass filter response (blue) in Fig. 4. The two-pass filter reduces the scalloping, but the width of the main lobe is decreased. This problem is remedied by a simple decrease in the filter length. In this case, a two-pass $N=10$ filter response (red) reduces aliasing and remains reasonably close to the desired cutoff wavenumber.

With a technique that is effectively able (with physical soundness) to separate the vortex scale (including its important asymmetries) from the subvortex scale, we will

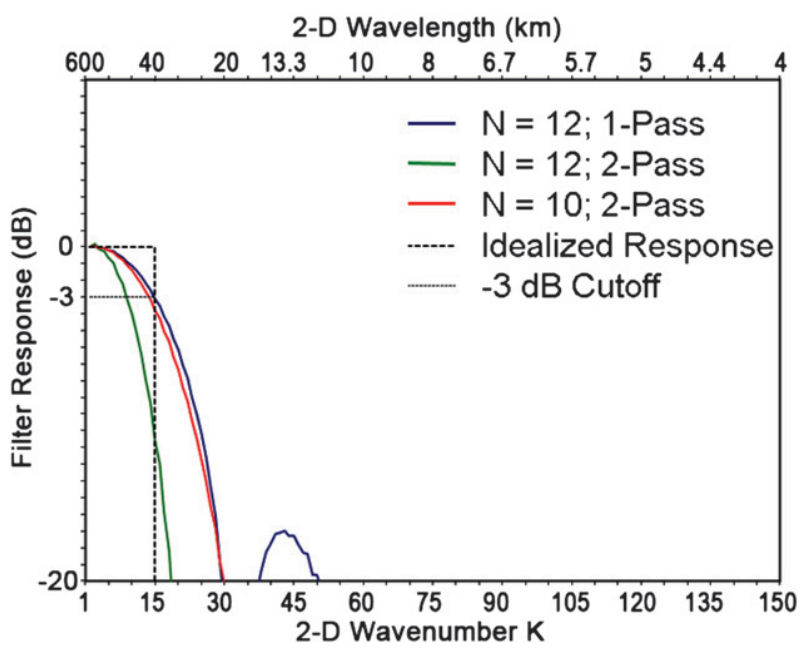

FIG. 4. Filter responses (dB) of the one-pass $N=12$ (blue), twopass $N=12$ (green), and two-pass $N=10$ (red) filters. The thick black dotted line is the idealized filter response. The thin black dotted line is the cutoff threshold; where this line intersects a filter response line is that filter's cutoff wavenumber. Note the sidelobe indicative of aliasing in the one-pass $N=12$ filter response.

now apply this technique to the M06-like simulations to quantify more robustly the vorticity and energy exchanges across that gap to better understand vortex growth and evolution in these simulations.

\section{Results}

This section first describes the development of the control experiment. Table 2 provides a timeline of the key events taking place during the 48-h simulation, with Fig. 5 providing a graphical representation of the vortex intensity during that period. Figures 6 and 7 show a time sequence of vorticity at $z=200 \mathrm{~m}$ and vertical velocity at $z=5 \mathrm{~km}$, respectively, for comparison with Fig. 5 and Table 2.

\section{a. Convective-scale structure}

A comparison of Figs. 6 and 7 shows that the dominant coherent structures are small-scale deep convective towers with strong cyclonic vorticity. These VHTs have typical updraft diameters less than $10 \mathrm{~km}$. Prior studies have concluded (Hendricks et al. 2004; Reasor et al. 2005; M06) that VHTs are the most important influence to the formation of the initial TC because VHTs create their own environment by radially trapping latent heat released from moist convection. This insulation preserves the vortex well after convection ceases by preventing it from shrinking vertically and reducing its angular velocity. In addition, VHTs sustain themselves from the destructive effects of downdrafts by consuming local CAPE, locally moistening the mid- to upper levels, and 
TABLE 2. Experiment A1: subjective description of system evolution. Please see Fig. 2 for a graphical representation of the intensity of the control simulation.

\begin{tabular}{|c|c|c|}
\hline Time (h) & Event & Description \\
\hline $0-2$ & Model initiation & Initial hot tower generated by incipient warm perturbation $50 \mathrm{~km}$ to west of vortex center. \\
\hline $2-13$ & Convection ceases & $\begin{array}{l}\text { Gravity wave interactions form a chain of weak amplitude vertical velocity perturbations. } \\
\text { These weak disturbances are advected by the tangential flow to the eastern side of the MCV. }\end{array}$ \\
\hline $13-18$ & $\begin{array}{l}\text { Secondary convection } \\
\text { initiates }\end{array}$ & $\begin{array}{l}\text { Gravity wave resonance causes localized peak vertical velocities to reach an apparent threshold } \\
\text { value of approximately } 15 \mathrm{~cm} \mathrm{~s}^{-1} \text {, at which time secondary convection commences. } \\
\text { Multiple subsequent VHTs begin to form a small }(\sim 40 \mathrm{~km}) \text { squall line along the local } \\
\text { horizontal shear vector. }\end{array}$ \\
\hline $18-26$ & Core organizes & $\begin{array}{l}\text { The individual VHT cores from the small squall line merge into a core vortex about } 20 \mathrm{~km} \\
\text { in diameter. Multiple smaller vortices begin to rotate around this core. }\end{array}$ \\
\hline $26-29$ & $\begin{array}{l}\text { Commencement of } \\
\text { system-scale growth }\end{array}$ & $\begin{array}{l}\text { The disturbance, now located to the north, expands outward until it intersects the rotational } \\
\text { axis of the MCV. The southern extent of the disturbance is pulled around this axis } \\
\text { causing rapid southward expansion of the initial asymmetry. }\end{array}$ \\
\hline $29-32$ & Core vortex strengthens & $\begin{array}{l}\text { Clear system-scale growth as core vortex is pulled around the MCV axis and smaller vortices } \\
\text { begin to spiral into the core. }\end{array}$ \\
\hline $32-40$ & System matures & $\begin{array}{l}\text { System is now comma shaped, with an intense cyclonic core and outward-spiraling tail } \\
\text { consisting of numerous vorticity couplets associated with strong VHT updrafts. }\end{array}$ \\
\hline $40-48$ & $\begin{array}{l}\text { Fully developed } \\
\text { spiral structure }\end{array}$ & $\begin{array}{l}\text { The system's moisture source is quickly being depleted and interior convection is stifled. } \\
\text { Outer bands also begin to show signs of weakening. A large }(\sim 100 \mathrm{~km}) \text { inner core persists, } \\
\text { while a smaller }(10-15 \mathrm{~km}) \text { stronger core rotates toroidally about the center. The cyclonic } \\
\text { core extends vertically from the surface to about } 9 \mathrm{~km} \text {. }\end{array}$ \\
\hline
\end{tabular}

diabatically merging with one another (Hendricks et al. 2004; M06).

A description of the thermodynamic structure of the primary vortex during the system merger provides a better understanding of the complexities of these vortex mergers. Figure 8 shows horizontal and vertical cross sections of relative vorticity and $\theta$ at $26 \mathrm{~h}$. Marked in Fig. 8 a by the sharp $\theta$ gradient starting at $x=290 \mathrm{~km}$ and $y=$ $310 \mathrm{~km}$, a front stretches southward along the eastern edge of a surface cold pool that surrounds a core of cyclonic vorticity. A long band of cyclonic vorticity collocated with this front feeds into the core vortex, consistent with the Reasor et al. (2005) description of a vorticity feeder band. Meanwhile, a deep column of positive vorticity in Fig. 8b extends upward from the cyclonic core seen in Fig. 8a and is accompanied by a sharp trough of warm $\theta$ values. The vorticity feeder band slopes over the surface cold pool to the east of this core, as indicated in Fig. 8 b by a westward tilt of cyclonic vorticity following $\theta=306 \mathrm{~K}$.

The vorticity feeder band feeds the core vortex by advecting into it positive vorticity generated within the vorticity feeder band itself. The positive vorticity stretching tendency shown in Fig. 9 (contours) indicates that this generation comes from vertical stretching of cyclonic vorticity. A line of ascent (Fig. 9, shaded) ahead of the cold pool stretches the cyclonic vorticity within the vorticity feeder band, strengthening it. A comparison of Figs. $8 \mathrm{a}$ and 9 reveals a complex interplay of three distinct subregions within this vorticity feeder band:
1) A primary vorticity generation region lies within the overlap between positive vertical velocity and vorticity stretching seen in Fig. 9.

2) An advective region stretches north-northwestward from this region, seen as an equally intense cyclonic segment of the vorticity feeder band in the vorticity field in Fig. 8a, but not in the stretching tendency field in Fig. 9. The lack of vorticity generation in this area suggests that this region is purely advective.

3) A turbulent convective region stretches westward from the generation region, spiraling outward from the core. Strong localized updrafts associated with highly turbulent vorticity dispersion characterize this

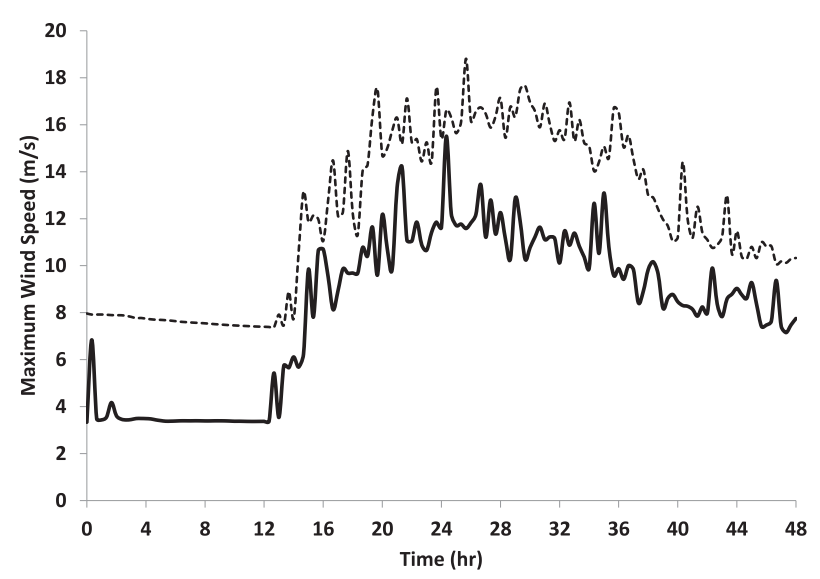

FIG. 5. Time series for the first $48 \mathrm{~h}$ of lowest-model-level maximum surface (solid) and domain-maximum (dashed) wind speed $\left(\mathrm{m} \mathrm{s}^{-1}\right)$ for the control run. 


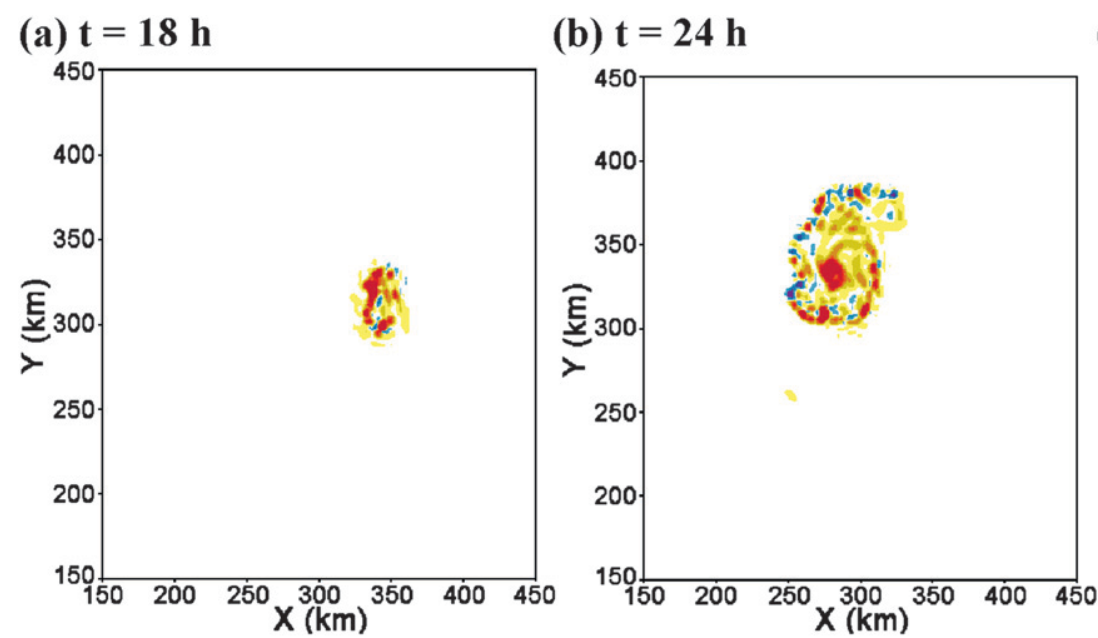

(c) $\mathrm{t}=26 \mathrm{~h}$

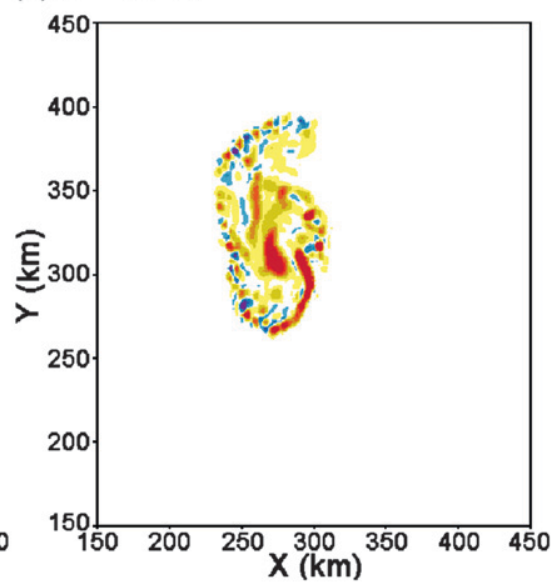

(e) $t=34 h$
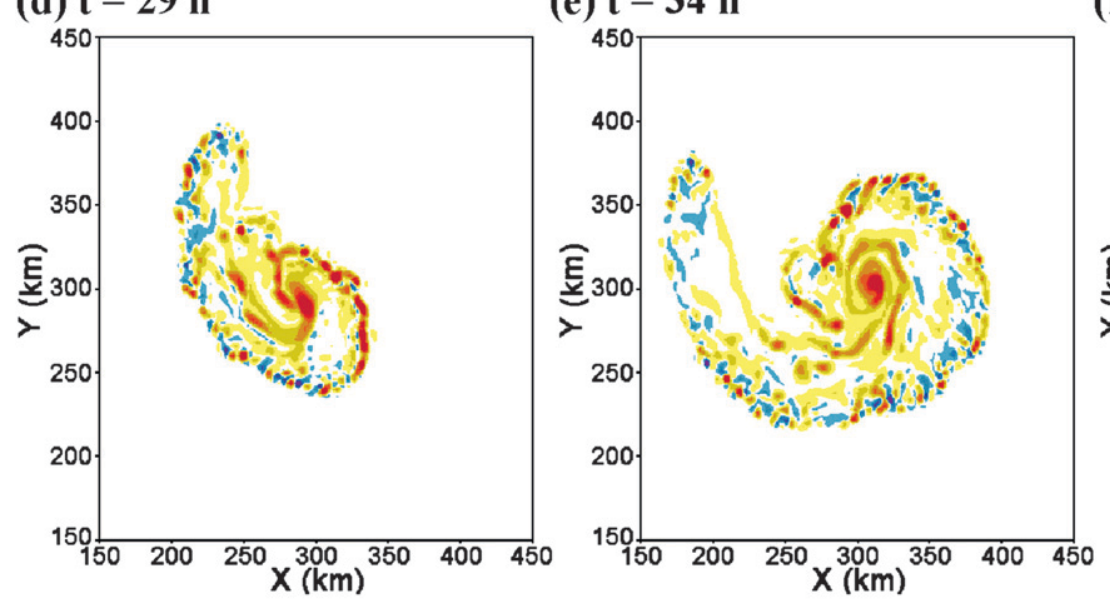

(f) $t=48 h$
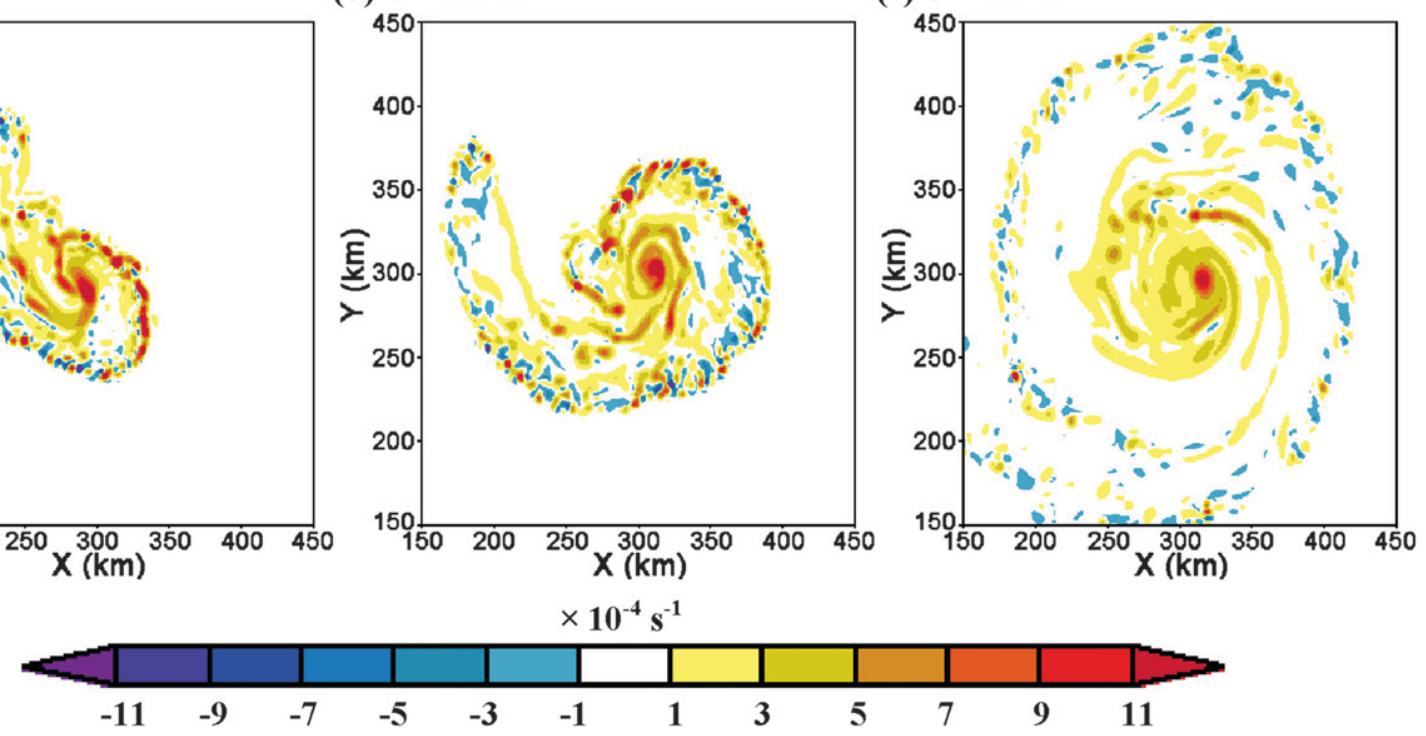

FIG. 6. Time series of near-surface $(z=200 \mathrm{~m})$ vorticity $\zeta\left(10^{-4} \mathrm{~s}^{-1}\right)$ at $t=(\mathrm{a}) 18$, (b) 24, (c) 26, (d) 29, (e) 34, and (f) $48 \mathrm{~h}$. Compare with key events listed in Table 2. Note: not full domain.

region. These updrafts form numerous small-scale vorticity couplets that spiral outward from the core (many of which are out of view; see Fig. 6b). The next section shows that at the system scale these couplets largely cancel each other through dissipation in a manner analogous to turbulent diffusion.

The complex evolution of small-scale features just described argues strongly for a full-scale vorticity budget. Further, given the complexity of these detailed structures, it is not immediately evident what will be the cascade of energy from the convective scale to the system-scale vortex, as newly defined here. Indeed, using the classic approach of symmetric-asymmetric separation, the largerscale asymmetric aspects of the vortex might easily overshadow the high-resolution details of convectivescale evolution. The next sections examine the systemscale development and quantify the cascade of energy that occurs between the two, using the new scale-separation method.

\section{b. System-scale development}

The spatial filter described in section 2 was created to separate the convective scale from the system scale. Filtering in this way has another advantage over other averages in that it allows the mean to evolve in time and in three-dimensional space, while producing no temporal phase response like that found in a time average. An analysis of the filtered fields at $z=200 \mathrm{~m}$ gives greater insight into the evolution of the surface vortex. Figure 10 


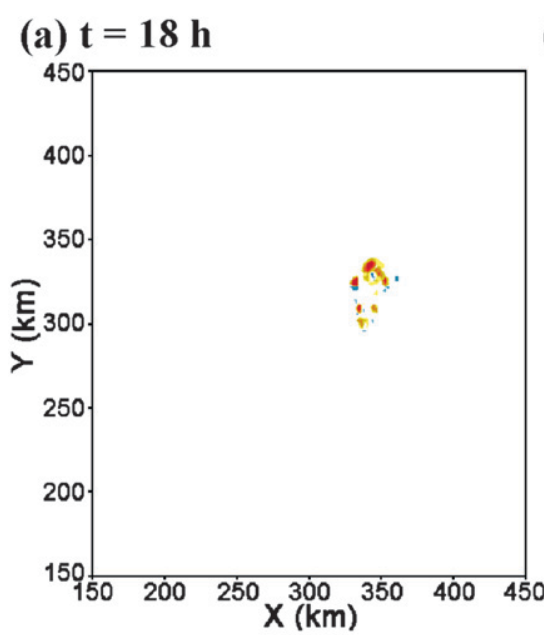

(b) $t=24 h$

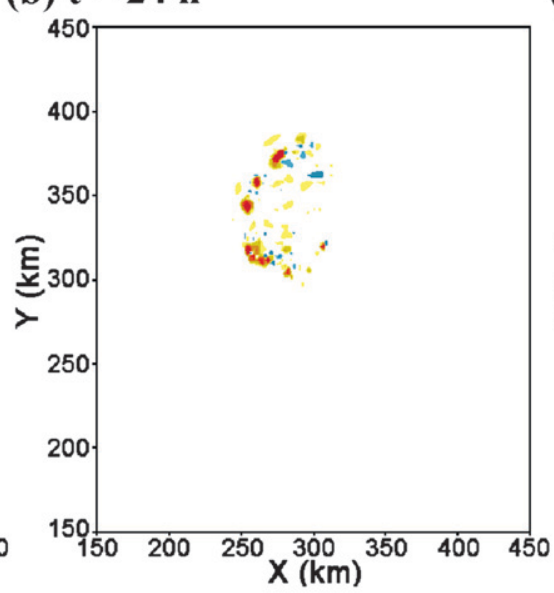

(c) $t=26 \mathrm{~h}$

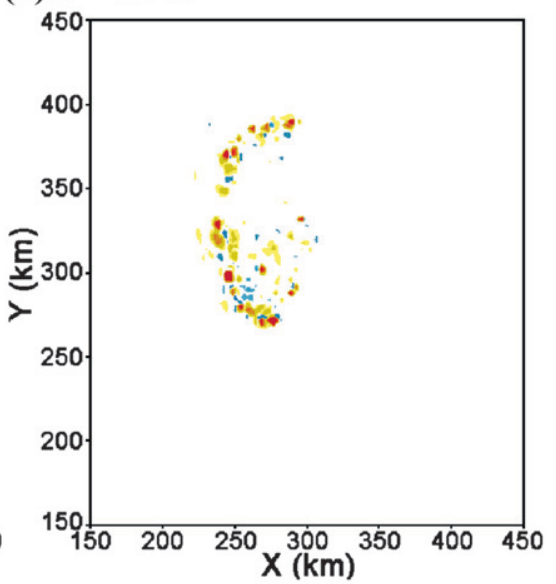

(d) $t=29 h$

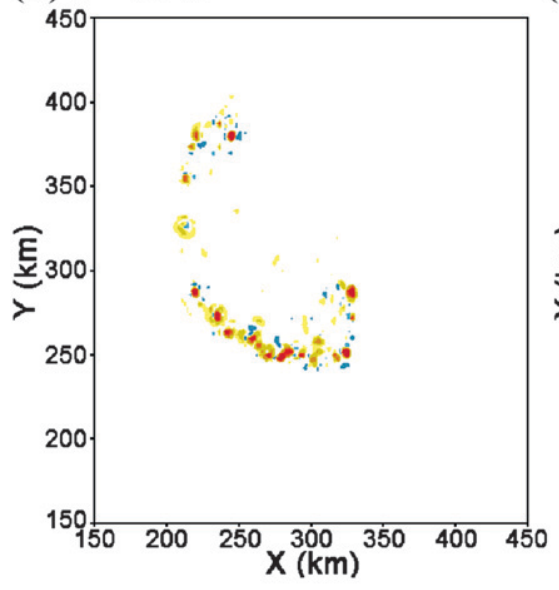

(e) $t=34 h$

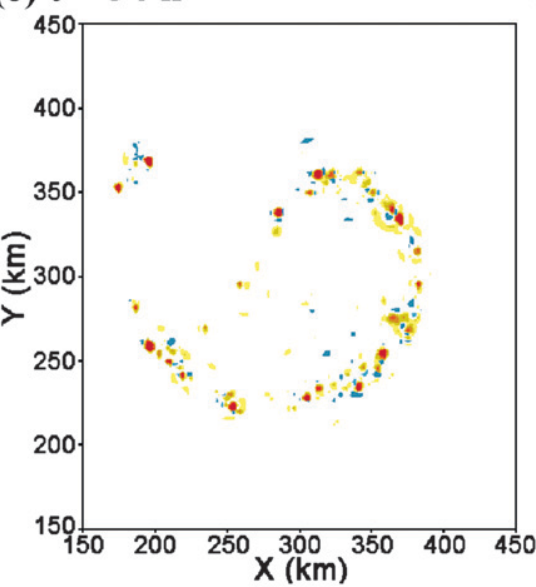

(f) $t=48 h$

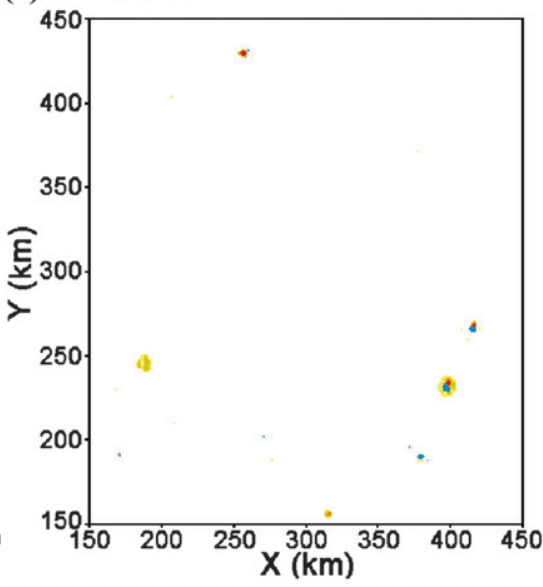

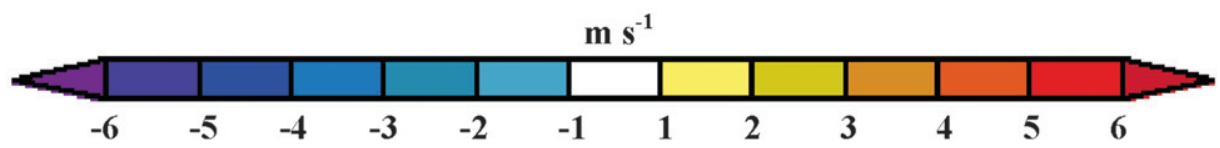

FIG. 7. Time series of vertical velocity $\left(\mathrm{m} \mathrm{s}^{-1}\right)$ at $z=5 \mathrm{~km}$ at $t=$ (a) 18 , (b) 24 , (c) 26, (d) 29, (e) 34, and (f) 48 h. Compare with key events listed in Table 2. Note: not full domain.

shows the spatially filtered fields corresponding to Fig. 6 . The foremost effect that these filtered fields show is the relatively insignificant role that the turbulent convective region plays on the system-scale vorticity, especially at later times. For instance, Fig. 6d shows a turbulent band of vorticity extending outward over $100 \mathrm{~km}$ to the northwest of center at 29 h. In Fig. 10d, this band has all but disappeared. Thus, one can argue that there is a net neutralization between positive and negative vorticity within the convective-scale vorticity couplets that dominate the turbulent convective region, given their lack of representation in the filtered vorticity field.

Another feature captured in the filtered 200 -m vorticity field is the apparent upscale cascade of vorticity occurring between 26 and 29 h (Figs. 10c and 10d). By 26 h, the system-scale vorticity has reached a maximum value of
$11 \times 10^{-4} \mathrm{~s}^{-1}$. At this time, it comes under the influence of a strong positive feeder band, which has intersected the rotational axis of the initial MCV. By $29 \mathrm{~h}$, the asymmetry has merged with the MCV core and the primary feeder band has stretched rapidly outwards to the east, and then to the north by $34 \mathrm{~h}$. At $48 \mathrm{~h}$, the system-scale vorticity field shows a fairly symmetric vortex. The MCV, which had an initial surface vorticity of about $0.5 \times$ $10^{-4} \mathrm{~s}^{-1}$, now has an estimated average vorticity of $2.0 \times$ $10^{-4} \mathrm{~s}^{-1}$, with a local maximum of $11 \times 10^{-4} \mathrm{~s}^{-1}$.

Analysis of the midlevel wind field shows that this intensification is not confined to the surface. Figure 11 shows the evolution of the filtered horizontal wind field at $z=5 \mathrm{~km}$. Of particular interest here is the intensification of the winds during the upscale cascade of vorticity at $26 \mathrm{~h}$. These winds reach a maximum magnitude 
(a)

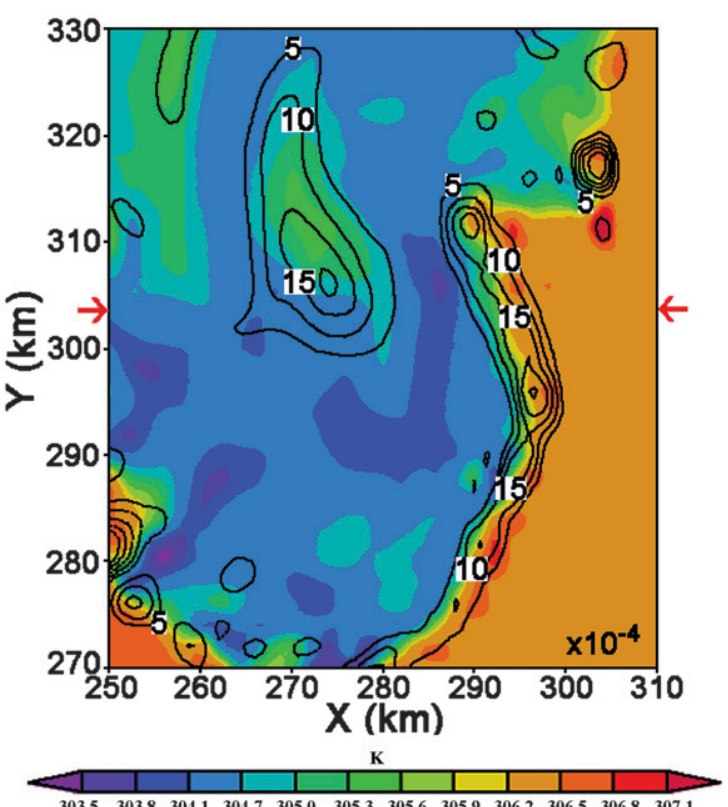

(b)

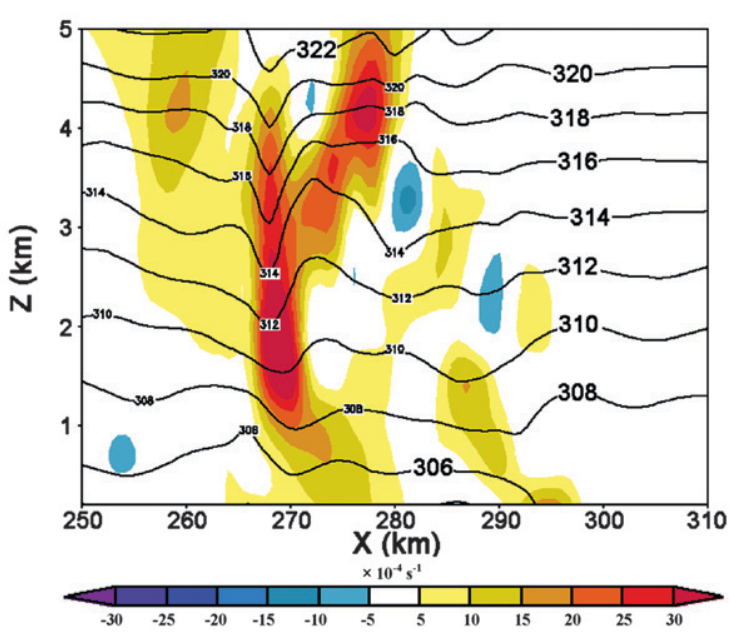

FIG. 8. Cross sections of primary vortex and vorticity "feeder band" structures at $t=26 \mathrm{~h}$. (a) Potential temperature (shaded; K), and vorticity (contours; $\mathrm{s}^{-1}$ ) at $z=200 \mathrm{~m}$. (b) Vorticity (shaded) and potential temperature (contours) at $y=304 \mathrm{~km}$. Zero contour omitted. Red arrows indicate location of vertical slice.

of $11 \mathrm{~m} \mathrm{~s}^{-1}$ around $34 \mathrm{~h}$. As early as $24 \mathrm{~h}$, the system's center of circulation is already under the influence of the initial asymmetry. At this time a double null in the wind field has begun to form, indicating a contention for control of the system's axis of rotation. By $26 \mathrm{~h}$, it is clear that the asymmetry has won the battle for control as a single null point remains approximately $20 \mathrm{~km}$ to the westsouthwest of the domain midpoint. This replacement center has a smaller radius and rotates toroidally about the location of the former center.

The prior discussions have described in qualitative terms the evolutions and interactions of both the

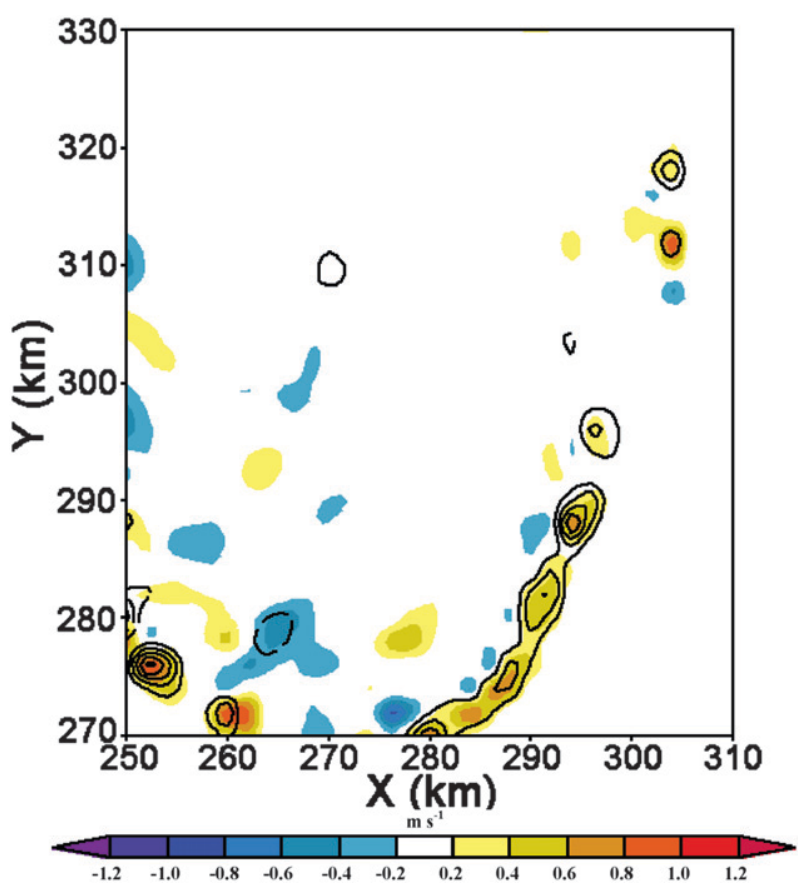

FIG. 9. As in Fig. 8a, but for vertical velocity (shaded, $\mathrm{m} \mathrm{s}^{-1}$ ) and vorticity stretching tendency (contours, interval $=1 \times 10^{-6} \mathrm{~s}^{-2}$ ). Negative contours are dashed and the zero contour is omitted.

convective-scale features and the system-scale features. However, those interactions are sufficiently complex that a quantification of the energy cascade and the contributions to that cascade are necessary to elucidate the amplification of the system scale, as shown in the next two sections.

\section{c. Vorticity spectrum analysis}

One way to examine the development of vorticity at different scales is to study the temporal evolution of the vorticity spectrum. Figure 12 is a Hovmöller diagram of the normalized power contributions for all 2D wavenumbers during the 48-h control simulation period, obtained by taking the FFT of the vorticity field at $z=200 \mathrm{~m}$. Black arrows on the time axis demark key structural events (Table 2). At early times, the only strong power signal exists at wavenumbers 3 and 4 . This corresponds to wavelengths between 150 and $200 \mathrm{~km}$, which are consistent with the scale of the initial MCV. This signal remains unchanged until about $16 \mathrm{~h}$ when two noteworthy events occur:

1) An emergence of high-wavenumber power centered at $K=75(\lambda=8 \mathrm{~km})$ that rapidly spreads to higher and lower wavenumbers.

2) An enhancement of the MCV spectral signal, associated with an increase in activity within the spectral gap between the MCV and convective-scale signals. 


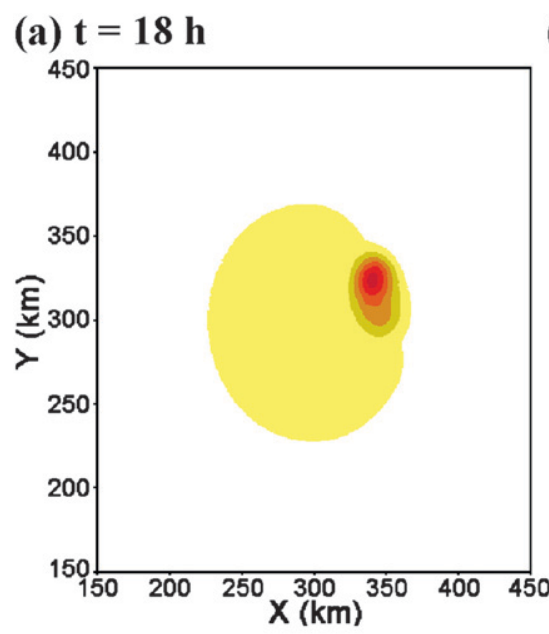

(b) $t=24 h$

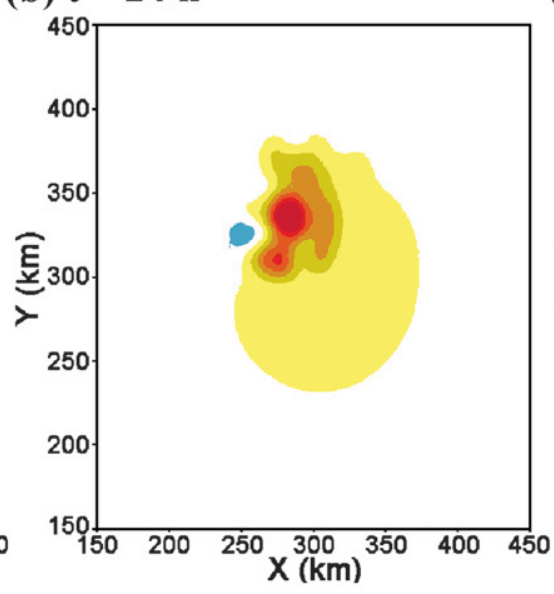

(c) $t=26 \mathrm{~h}$

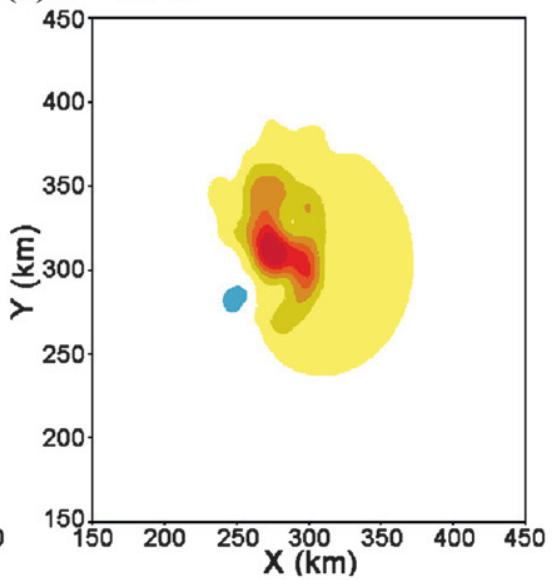

(d) $t=29 h$

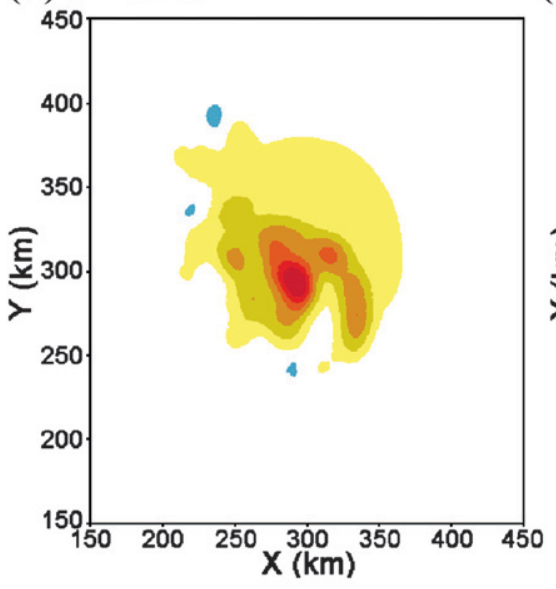

(e) $t=34 h$

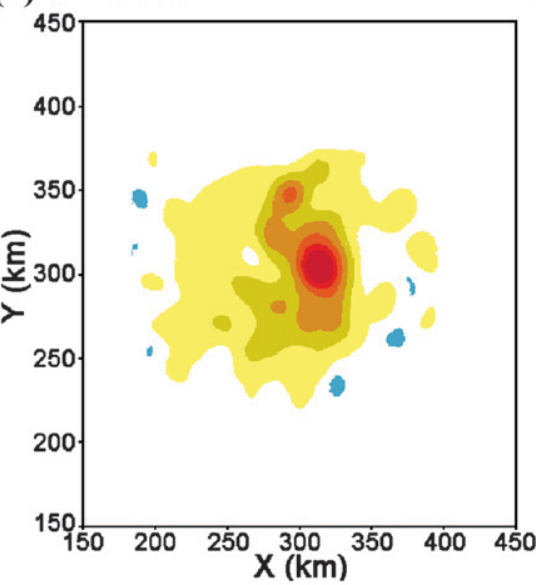

(f) $t=48 h$

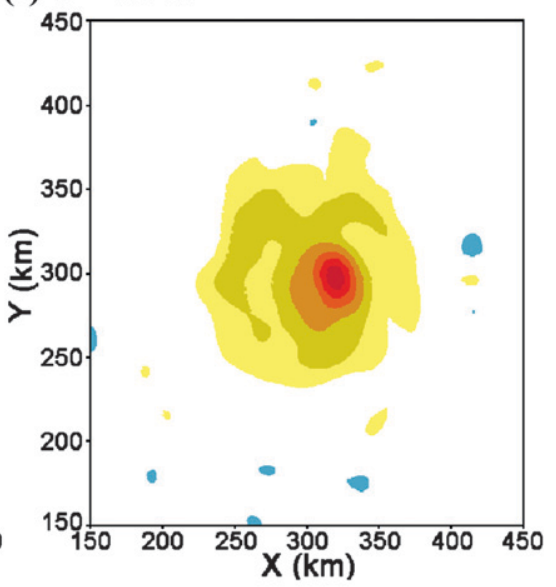

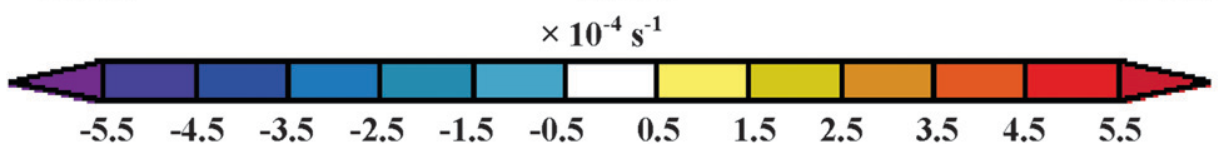

FIG. 10. Time series of near-surface $(z=200 \mathrm{~m})$ spatially filtered vorticity $\bar{\zeta}\left(10^{-4} \mathrm{~s}^{-1}\right)$ at $t=$ (a) 18 , (b) 24 , (c) 26 , (d) 29 , (e) 34 , and (f) 48 h. Compare with Fig. 8.

There are at least two "middle scale" formations that appear at $K=8(\lambda=75 \mathrm{~km})$ and $K=12(\lambda=50 \mathrm{~km})$. At $26 \mathrm{~h}$, this middle-scale power band appears to actually feed into and augment the MCV signal.

The physical interpretation of (1) is that upon the initiation of convection, localized vorticity centers with scales of about $8 \mathrm{~km}$ are expanding upscale and diffusing downscale. Judging from the quick expansion toward low wavenumbers, rapid upscale growth seems to be occurring because of a localized conglomeration of vorticity centers in the area of convection. Throughout the entirety of the simulation, this upscale growth is continuous in spectral space for wavenumbers greater than $K=15$ or for wavelengths less than $40 \mathrm{~km}$.
The explanation of (2) is less apparent. While an enhancement of the initial MCV is clearly apparent, the origin of these middle-scale formations is unclear. These formations exhibit similar characteristics to aliasinginduced side lobes. That is, they initially seem to be projections of lower-wavenumber energy onto higher wavenumbers, possibly artifacts of wavenumber binning within the FFT scheme. However, these middle-scale lobes do not appear everywhere and seem to be related to major pulses in high-wavenumber power. The first two lobes seem to reach maximum intensity at 18 and $25 \mathrm{~h}$, in very close temporal proximity to the key events described in Table 2 (demarked in Fig. 12 by black arrows on the time axis). All of these events seem to be accompanied loosely by a successive flow and then ebb 


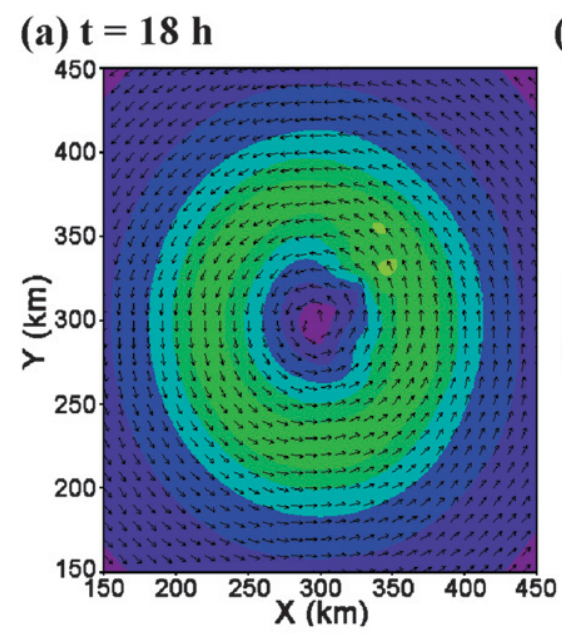

(b) $t=24 h$

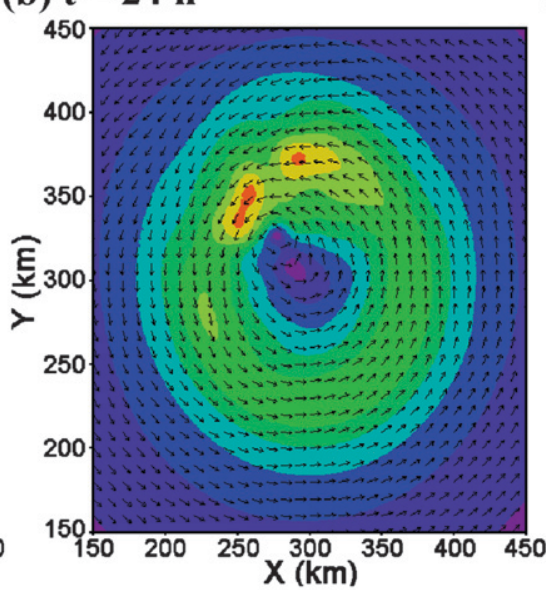

(c) $t=26 \mathrm{~h}$

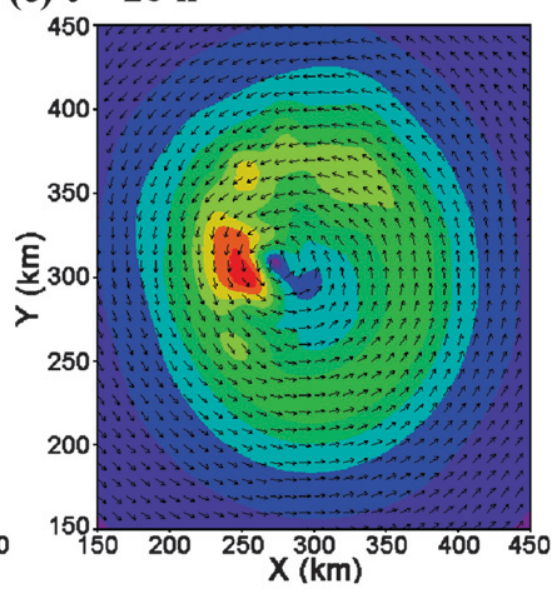

(d) $t=29 h$

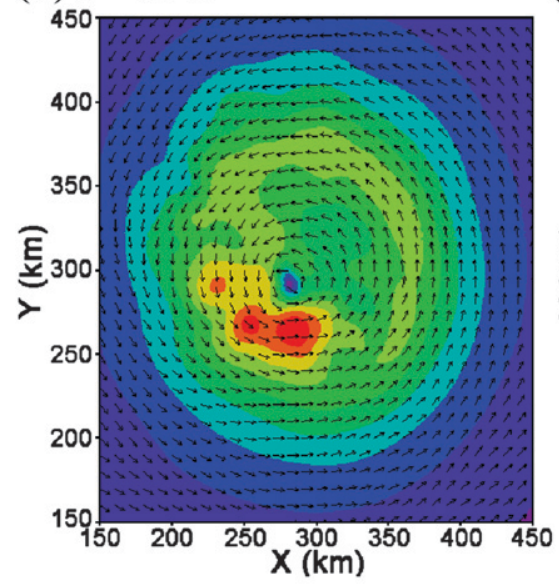

(e) $t=34 \mathrm{~h}$

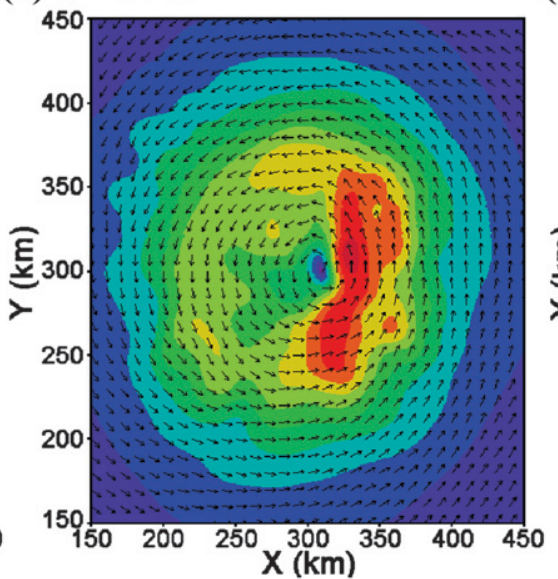

(f) $t=48 h$

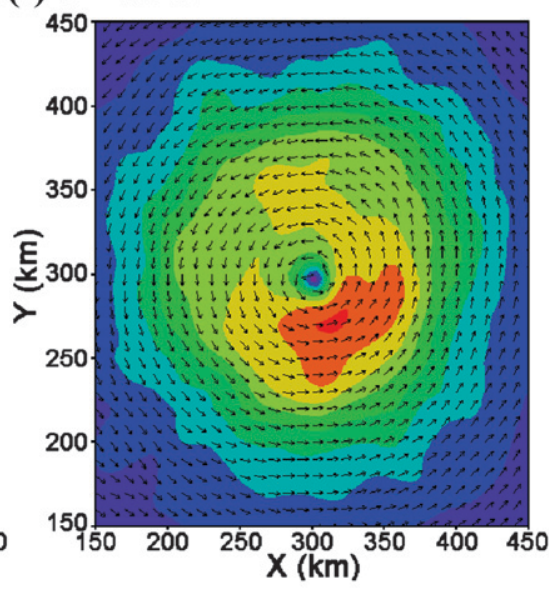

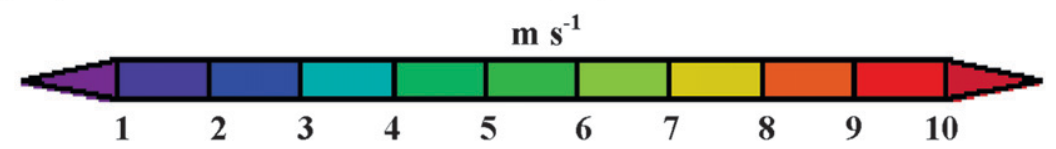

FIG. 11. Time series of spatially filtered 5-km horizontal wind $\left(\mathrm{m} \mathrm{s}^{-1}\right)$ at $t=$ (a) 18, (b) 24, (c) 26, (d) 29, (e) 34 , and (f) 48 h. Magnitudes are shaded; vectors indicate direction. Note the motion and decrease in radius of the center of rotation before and after $t=26 \mathrm{~h}$.

of high-wavenumber energy into low-wavenumber energy. This suggests that these middle-scale phenomena may not be artifacts of the FFT scheme, but instead a spectral representation of an incremental upscale cascade of energy during major vortex merger processes. The fact that the existence of these phenomena appears to correspond with significant increases in power and/ or widening of the MCV spectral signal supports this argument. For instance, at $26 \mathrm{~h}$, the middle lobe appears to merge briefly with the MCV spectral signal, while the MCV signal itself appears to expand into higher wavenumbers toward the middle-scale pulse.

Ritchie and Holland (1997) studied this type of upscale cascade of vorticity and pointed to scale interactions between midlevel mesoscale vortices and their synoptic-scale environment as a key factor in TC formation. They found that "environmental and mesoscale dynamics mutually enhance each other in a cooperative interaction during cyclone formation." Within the numerical simulations presented in this study, a similar phenomenon occurs between the mesoscale and convective scales. Experiments performed without any initial MCV (not shown) highlight the absolute necessity of this mesoscale support in the organization of the incipient surface vortex (verified by BE97).

The upscale cascade of rotational energy inspires a deeper investigation into the dynamical evolution of system-scale vorticity. Figure 12 hints that system-scale vorticity increases during the course of the control experiment and appears to do so over discrete intervals. 


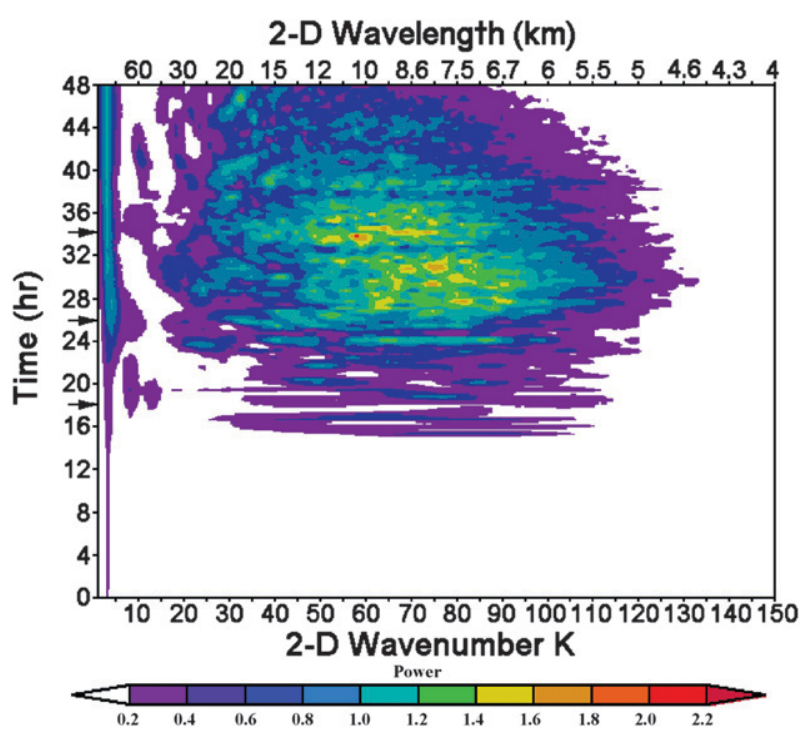

FIG. 12. Hovmöller diagram depicting temporal evolution of the power spectrum of near-surface $(200 \mathrm{~m})$ vorticity. Shaded values represent normalized power at horizontal wavenumber values ranging from 1 (long wave) to 150 (short wave). Black arrows on the time axis demark the events noted in Table 2. Note that the temporal average of this figure results in Fig. 4.

Leaving spectral space for the time being, a spatial aggregate of system-scale vorticity can also quantify this growth, as discussed next.

\section{d. Circulation tendency}

Figure 13 shows a vertical Hovmöller diagram depicting the evolution of circulation, calculated over a 50-km-radius circle at domain center, within the RMW of the initial MCV. At the onset, the cyclonic circulation signal due to the MCV extends vertically from the surface to about $8 \mathrm{~km}$ and peaks at the level of maximum winds at $z=3.5 \mathrm{~km}$ (refer to the turquoise-to-green shading on the left side of Fig. 13). Before the secondary convection event starting at $13 \mathrm{~h}$ (Table 2) there is a slight weakening of this initial MCV circulation. Sensitivity experiments with no initial convection (not shown) confirm that this weakening is due entirely to model adjustments of the analytic initial gradient wind-balanced MCV to the discretized equations used within WRF. At $18 \mathrm{~h}$, a clear upward expansion of the cyclonic circulation from 8 to $10 \mathrm{~km}$ occurs without obvious immediate intensification thereafter. However, at $25 \mathrm{~h}$, the cyclonic circulation of the MCV below 9 km clearly enhances, and at $29 \mathrm{~h}$ it expands vertically once more to just under $12 \mathrm{~km}$; both of these events closely coincide with the convective-scale mergers described in Table 2. After $29 \mathrm{~h}$ the MCV cyclonic circulation intensifies, starting above the level of maximum winds then later extending

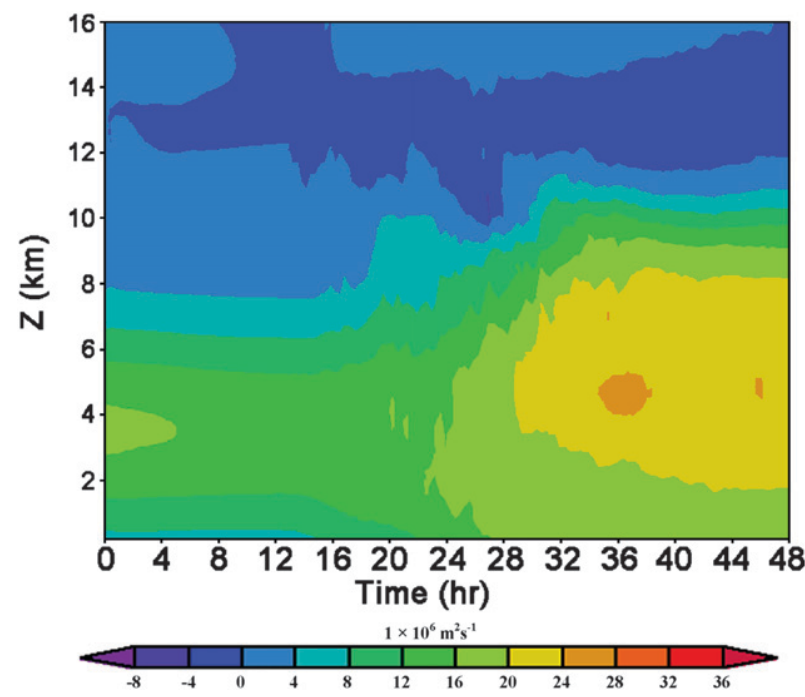

FIG. 13. Vertical Hovmöller diagram of circulation $\left(\mathrm{m}^{2} \mathrm{~s}^{-1}\right)$ at a radius of $50 \mathrm{~km}$ from domain center. Note the enhancement of the midlevel circulation beginning around $t=25 \mathrm{~h}$, during the growth of the system scale as described in Table 2 .

slowly toward the surface until about $40 \mathrm{~h}$, when it becomes relatively constant.

The rate of change of circulation with time helps to quantify and understand the processes responsible for these changes in circulation:

$$
\frac{\partial \Gamma}{\partial t} \cong \pi R_{C}^{2}\left\{\frac{\partial \zeta}{\partial t}\right\}_{A}
$$

where $R_{c}$ is the circle radius (here set to $50 \mathrm{~km}$ ) and curly brackets represent the area average over that circle. As in (8) and (9), the individual contributions to $\partial \Gamma / \partial t$ from vorticity stretching and tilting are as follows:

$$
\begin{gathered}
\left.\frac{\partial \Gamma}{\partial t}\right|_{\mathrm{DIV}} \cong \pi R_{C}^{2}\left\{\left.\frac{\partial \zeta}{\partial t}\right|_{\mathrm{DIV}}\right\}_{A}, \\
\left.\frac{\partial \Gamma}{\partial t}\right|_{\mathrm{TT}} \cong \pi R_{C}^{2}\left\{\left.\frac{\partial \zeta}{\partial t}\right|_{\mathrm{TT}}\right\}_{A} .
\end{gathered}
$$

Figure 14 shows Hovmöller diagrams of the vertical structure of the stretching and tilting components of circulation tendency as well as radial and vertical advection contributions. Figure 14a confirms that the main supplier of cyclonic circulation is the net stretching tendency, which is generally positive, especially below $8 \mathrm{~km}$. Note that the majority of tilting production (Fig. $14 \mathrm{~b}$ ), which is largely negative at upper and lower levels and positive at midlevels, is associated with an opposing vertical advection tendency (Fig. 14d). This suggests that the anticyclonic vorticity generated by tilting advects upward, away from the location of its creation. Radial vorticity advection (Fig. 14c) exhibits rapid temporal 

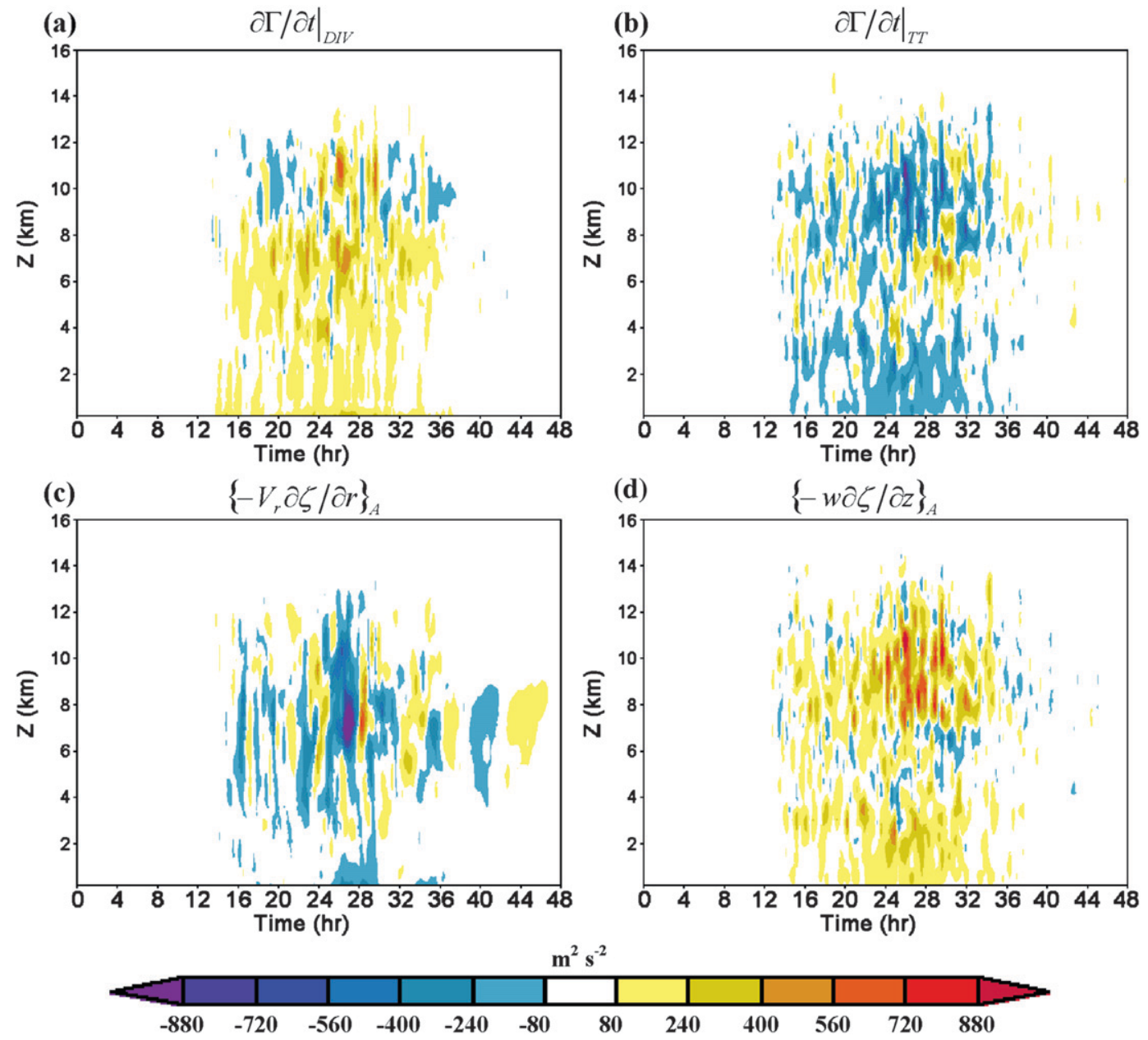

FIG. 14. Vertical Hovmöller diagrams of circulation tendencies $\left(\mathrm{m}^{2} \mathrm{~s}^{-2}\right)$ at a radius of $50 \mathrm{~km}$ from domain center including circulation tendency due to (a) vorticity stretching, (b) vorticity tilting, (c) radial vorticity advection, and (d) vertical vorticity advection.

fluctuations between positive and negative tendencies, associated with short-term fluctuations in the centroid of the vortex and its representation on the discrete grid. ${ }^{4}$ Regardless, the sum (not shown) of the tendencies in Fig. 14 indicates a net positive circulation tendency primarily from 2 to $8 \mathrm{~km}$, which is broadly consistent with the circulation increases in Fig. 13.

The primary vorticity production mechanisms now identified, a reassessment of (11) and (12) quantifies how

\footnotetext{
${ }^{4}$ This also is likely a by-product of calculating circulation over a circle that is fixed to the center of the domain. If the circle were allowed to follow the centroid of the vortex, the rapid fluctuations seen in the advection term would likely be much more muted. Such a methodology for calculating circulation would better allow meaning to be assigned to the apparent anticorrelation between the radial advection term and the divergence terms, but is outside the scope of this study.
}

much of this contribution comes from the convective scale. As a consequence of the areal averages taken within the filtered terms themselves, $\{\partial \bar{\zeta} / \partial t\}_{A} \approx\{\partial \zeta / \partial t\}_{A}$ when $R_{C}$ is greater than the filter length. Thus, (16) and (17) may be rewritten as follows:

$$
\begin{aligned}
\left.\frac{\partial \Gamma}{\partial t}\right|_{\mathrm{DIV}} & \cong \pi R_{C}^{2}\left\{\left.\frac{\partial \zeta}{\partial t}\right|_{\mathrm{DIV}}\right\}_{A} \approx \pi R_{C}^{2}\left\{\left.\frac{\overline{\partial \zeta}}{\partial t}\right|_{\mathrm{DIV}}\right\}_{A} \\
& =\pi R_{C}^{2}\{\mathrm{MMD}+\mathrm{MED}+\mathrm{EMD}+\mathrm{EED}\}_{A},
\end{aligned}
$$

$$
\begin{aligned}
\left.\frac{\partial \Gamma}{\partial t}\right|_{\mathrm{TT}} \cong \pi R_{C}^{2}\left\{\left.\frac{\partial \zeta}{\partial t}\right|_{\mathrm{TT}}\right\}_{A} \approx \pi R_{C}^{2}\left\{\left.\frac{\overline{\partial \zeta}}{\partial t}\right|_{\mathrm{TT}}\right\}_{A} \\
=\pi R_{C}^{2}\{\mathrm{MMT}+\mathrm{MET}+\mathrm{EMT}+\mathrm{EET}\}_{A}
\end{aligned}
$$

The MM and EE terms in these equations turn out to be at least an order of magnitude larger than the ME or EM 

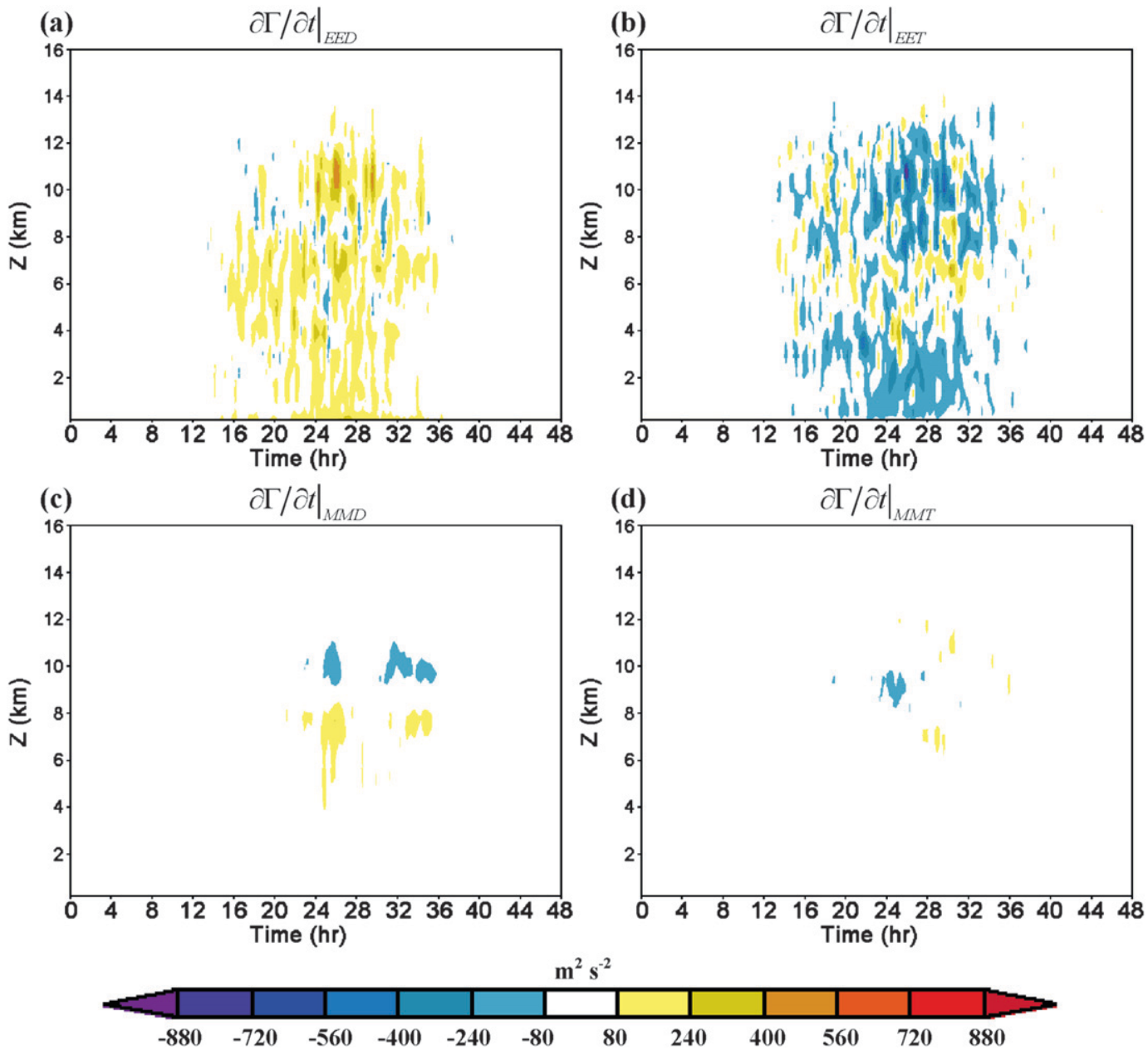

FIG. 15. Vertical Hovmöller diagrams of circulation tendencies $\left(\mathrm{m}^{2} \mathrm{~s}^{-2}\right)$ at a radius of $50 \mathrm{~km}$ from domain center including component of circulation tendency due to (a) eddy stretching of eddy vorticity, (b) eddy tilting of eddy horizontal vorticity, (c) mean stretching of mean vorticity, and (d) mean tilting of mean horizontal vorticity.

terms, so the four important scale-dependent tendency terms become

$$
\begin{aligned}
\left.\frac{\partial \Gamma}{\partial t}\right|_{\mathrm{MMD}} & =\pi R_{C}^{2}\{\mathrm{MMD}\}_{A} \\
& =\pi R_{C}^{2}\left\{-\overline{(\bar{\zeta}+f)\left(\frac{\partial \bar{u}}{\partial x}+\frac{\partial \bar{v}}{\partial y}\right)}\right\}_{A}, \\
\left.\frac{\partial \Gamma}{\partial t}\right|_{\mathrm{EED}} & =\pi R_{C}^{2}\{\mathrm{EED}\}_{A}=\pi R_{C}^{2}\left\{-\overline{\zeta^{\prime}\left(\frac{\partial u^{\prime}}{\partial x}+\frac{\partial v^{\prime}}{\partial y}\right)}\right\}_{A},
\end{aligned}
$$

$$
\begin{aligned}
\left.\frac{\partial \Gamma}{\partial t}\right|_{\mathrm{MMT}} & =\pi R_{C}^{2}\{\mathrm{MMT}\}_{A} \\
& =\pi R_{C}^{2}\left\{-\overline{\left(\frac{\partial \bar{w}}{\partial x} \frac{\partial \bar{v}}{\partial z}-\frac{\partial \bar{w}}{\partial y} \frac{\partial \bar{u}}{\partial z}\right)}\right\}_{A},
\end{aligned}
$$

$$
\begin{aligned}
\left.\frac{\partial \Gamma}{\partial t}\right|_{\mathrm{EET}} & =\pi R_{C}^{2}\{\mathrm{EET}\}_{A} \\
& =\pi R_{C}^{2}\left\{-\overline{\left(\frac{\partial w^{\prime}}{\partial x} \frac{\partial v^{\prime}}{\partial z}-\frac{\partial w^{\prime}}{\partial y} \frac{\partial u^{\prime}}{\partial z}\right)}\right\}_{A} .
\end{aligned}
$$

Figure 15 shows relative contributions by these four scale-dependent circulation tendency terms. The key observation here is the magnitude discrepancy between system-scale influences and convective-scale influences. Within the control simulation, in both the stretching and tilting terms, the convective-scale contributions are most important in the generation/destruction of the systemscale circulation (Figs. 15a,b). Weaker system-scale tendencies (Figs. 15c,d) later become apparent owing to the development of an upper-level toroidal circulation (not shown).

Referring to Fig. 15a, the EED circulation tendency term is the main source of cyclonic vorticity production 
within the control vortex. The EED term is positive at nearly every level below $12 \mathrm{~km}$ and at every time, yet the convective-scale divergence pattern (not shown) varies in the vertical from largely divergent at the surface and above $9 \mathrm{~km}$ to largely convergent from 2 to $8 \mathrm{~km}$. This divergence pattern likely arises from conservation of angular momentum: there is stretching of positive perturbation vorticity in association with strong positive updraft accelerations. Above $9 \mathrm{~km}$, enhanced perturbation divergence at the equilibrium level weakens perturbation anticyclonic vorticity. Similarly, from the surface to $2 \mathrm{~km}$, convective-scale divergence horizontally expands anticyclonic vorticity, most likely associated with rain-induced downdrafts, but more research is required to ascertain the details.

The dominance of the EED term contradicts the argument that a net system-scale convergence of vorticity is most responsible for the spinup of the initial MCV. The positive feedback mechanism occurring within the divergence term seems to be the key to the dominance of the EED term. Although the initial MCV is perhaps the early source of cyclonic vorticity, once this cyclonic seed is planted the convective-scale positive feedback mechanism takes control. The trigger for this mechanism comes in the form of strong convective-scale convergence associated with intense CAPE-fueled updrafts. The positive feedback cycle ceases with the consumption of this CAPE.

\section{e. Sensitivity experiments}

Sensitivity experiments allow for examination of the effect of two factors on the development of system-scale vorticity: (i) enhancing low-level moisture within the incipient MCV as in M06 (experiment A2) and (ii) introducing multiple initial temperature perturbations (experiment B1). Experiment B2 is a combination of these two factors (see Table 1).

Initial moisture content had the most significant impact on system evolution, expediting the onset of secondary convection and increasing the magnitude and horizontal scale of the final vortex. Multiple perturbations slightly enhanced the vortex, but with only a small acceleration in the initiation of secondary convection. In each of these sensitivity experiments, the upscale cascade of vorticity coincided with the onset of secondary convection, which makes experiment $\mathrm{A} 1$ somewhat different from the other simulations, in that it had a clear distinction between system- and convective-scale events.

Figure 16 provides an overview of the spectral (left) and circulation (right) evolution within each of the sensitivity experiments. Figures $16 \mathrm{a}$ and $16 \mathrm{c}$ indicate that there is an accelerated development within the two moist simulations (A2 and B2). The vorticity power spectra of the moist experiments (Figs. 16a,c, left) show a convective signal that is wider and stronger than those in experiments A1 and B1. Meanwhile, the rapid upscale growth within the moist simulations seems to enhance the powercascade mechanism described in section $4 \mathrm{c}$.

In each of these experiments a growth of the middleto upper-level circulation closely follows an initial strengthening of the near-surface circulation (Fig. 16, right). From a standpoint of "top down" versus "bottom up" development, this quick transition from surface development upward may not in itself justify that this is a bottom-up development; however, it does at least preclude the possibility that these vortices grew via a stratiform precipitation-induced top-down mechanism. Further simulations using boundary layer and surface schemes are needed to confirm whether the mechanism described here is consistent with that described by M06 but are beyond the scope of this study.

Figure 17 depicts the MMD and EED circulation tendency terms for each of the three sensitivity experiments. Once again, the multiple perturbation simulation in Fig. $17 \mathrm{~b}$ differs little from the control; however, the moist experiments see a distinct enhancement in both divergence terms. Most notably, the MMD term greatly increases in magnitude. However, a similar enhancement of the EED term especially evident at upper levels accompanies this enhancement of the MMD term. This upperlevel generation of vorticity explains the rapid vertical growth of the circulations at $8 \mathrm{~h}$ within experiments $\mathrm{A} 2$ and B2 (Figs. 16a,c, right).

In summary, all four experiments evolved in a similar manner, yet had subtle but important differences in the development of the system-scale vortex. Specifically, these were (i) the lead time until secondary convection initiated growth, (ii) the symmetry within the initial development phase, and (iii) the maximum magnitude of the final circulation.

\section{Concluding summary}

A spatial filter, in which the desired filter length was determined via spectral analysis of the vorticity field, separated variables into system-scale means and convective-scale perturbations. This separation was a more physically sound separation than the conventional symmetric-asymmetric approach, given the inherently asymmetric structure of the vortex during and after genesis. Figure 18 summarizes the evolution of the systemscale vorticity field, highlighting these asymmetries. Thinking of these asymmetries in terms of spatial scale the analysis of prior TC genesis studies in a new context, as summarized next.

Hendricks et al. (2004) and M06 put forth the argument that VHTs contribute to an upscale organization 
(a) Expt. A2: Power

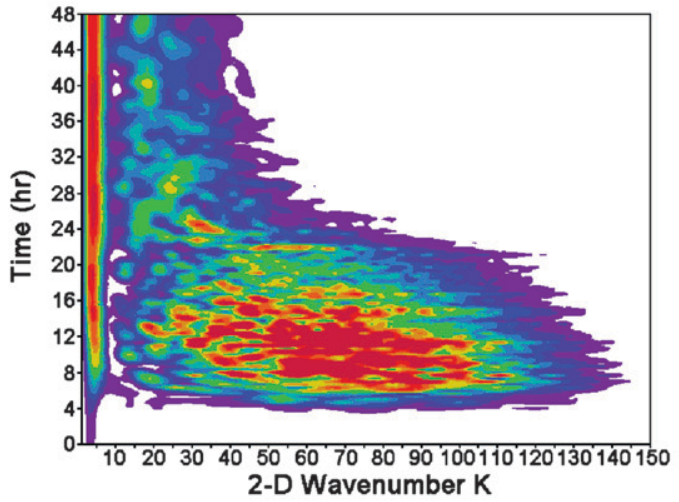

(b) Expt. B1: Power

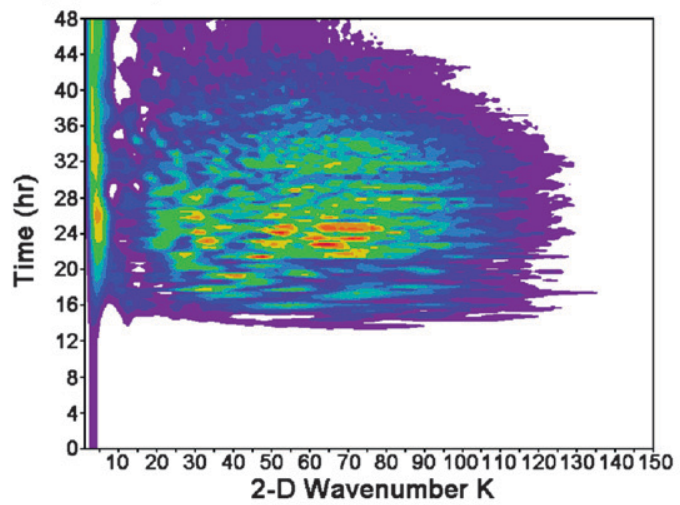

(c) Expt. B2: Power

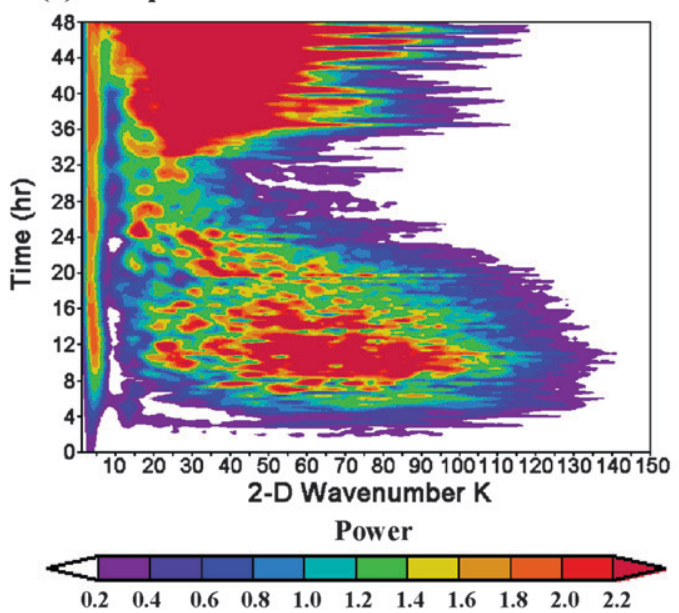

Circulation

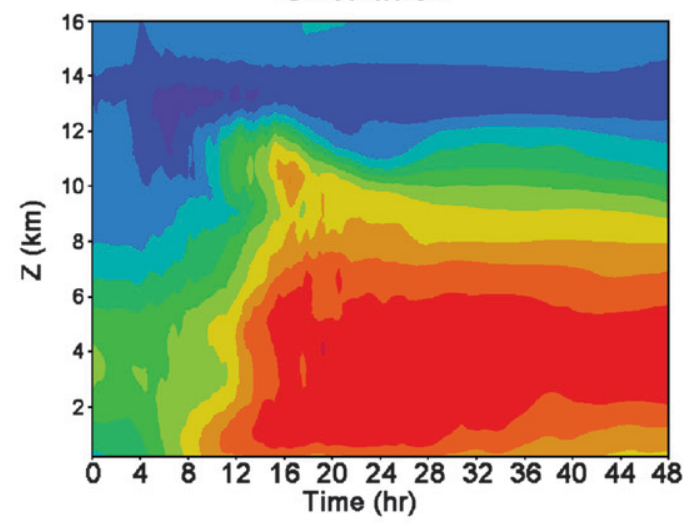

Circulation

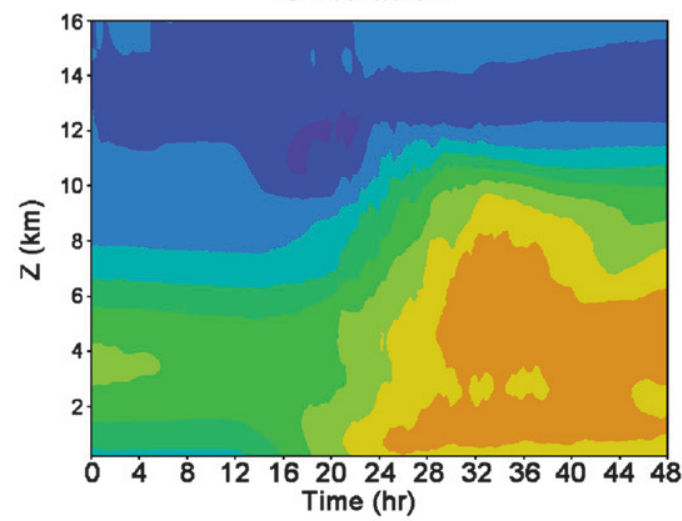

Circulation

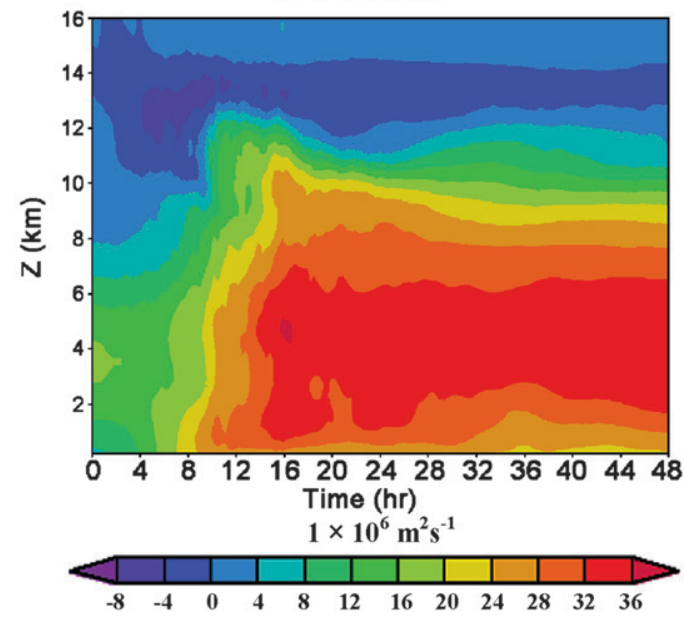

FIG. 16. Vertical Hovmöller diagrams of near-surface $(200 \mathrm{~m})$ (left) vorticity power spectra, as in Fig. 13, and (right) circulation, as in Fig. 14, for experiments (a) A2, (b) B1, and (c) B2.

of the pre-WISHE surface vortex. This argument motivated the use of three-dimensional numerical simulations to assess the explicit role of small-scale convection on the development of system-scale vorticity within an embryonic midlevel vortex. Applying a physically meaningful filter and incorporating the newly defined systemscale and convective-scale terms into the vorticity tendency equation assisted in the analysis of their relative contributions to the mean vorticity evolution. A summary of the primary findings of this analysis is as follows:

1) Convective-scale stretching of VHT-generated cyclonic vorticity plays the dominant role in the spinup of system-scale vorticity. 
(a) Expt. A2: $\quad \partial \Gamma /\left.\partial t\right|_{M M D}$

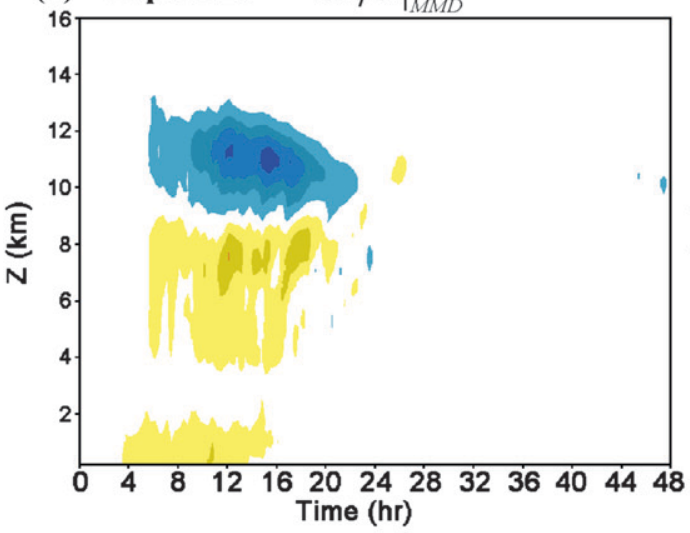

(b) Expt. B1: $\quad \partial \Gamma /\left.\partial t\right|_{M M D}$

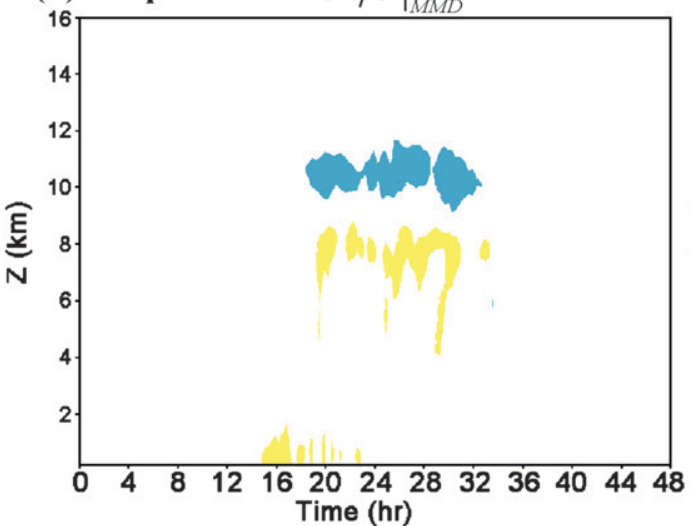

(c) Expt. B2: $\quad \partial \Gamma /\left.\partial t\right|_{M M D}$

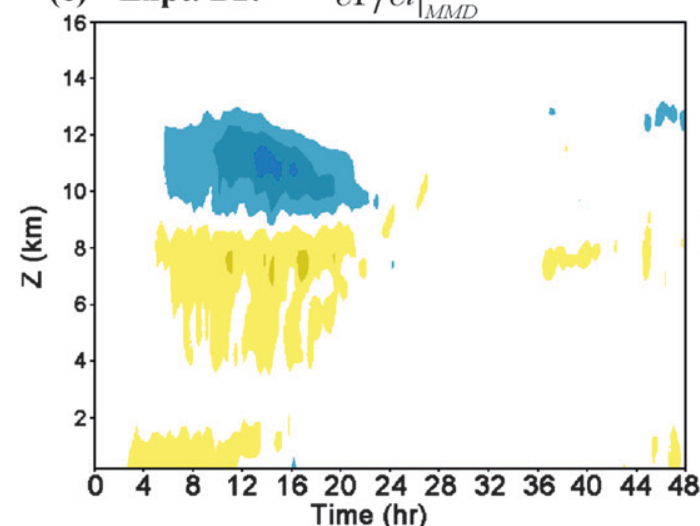

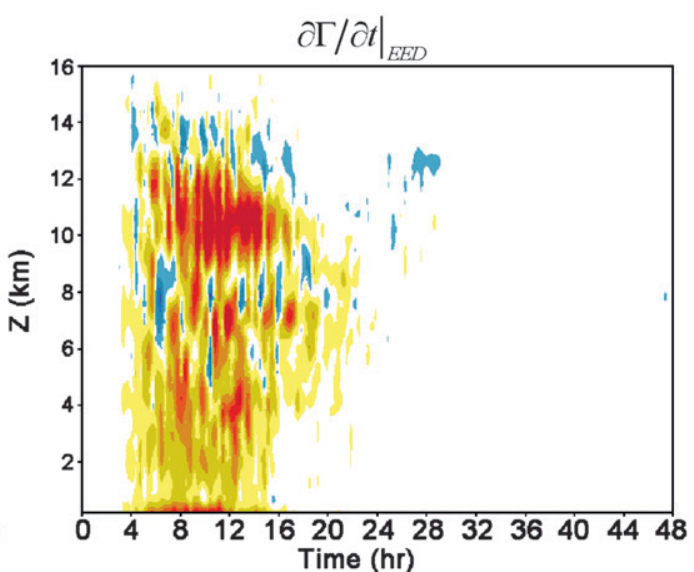

$\partial \Gamma /\left.\partial t\right|_{E E D}$

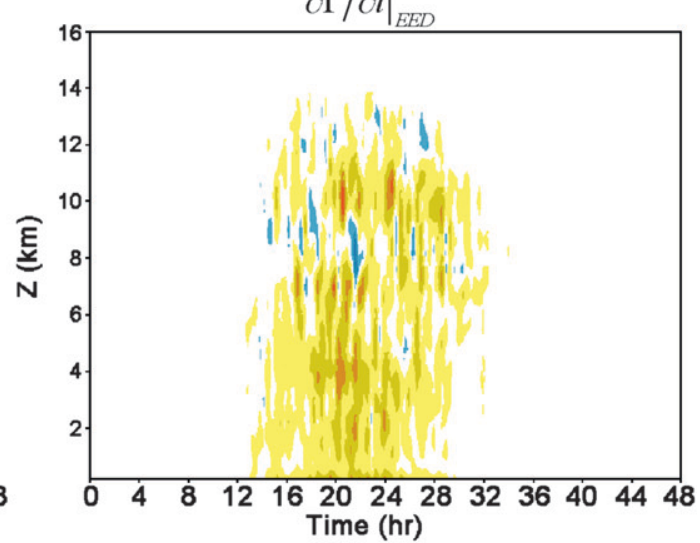

$\partial \Gamma /\left.\partial t\right|_{E E D}$

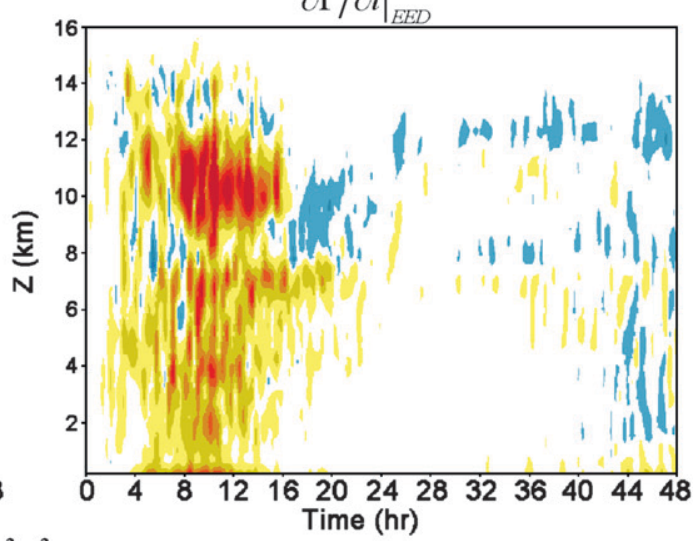

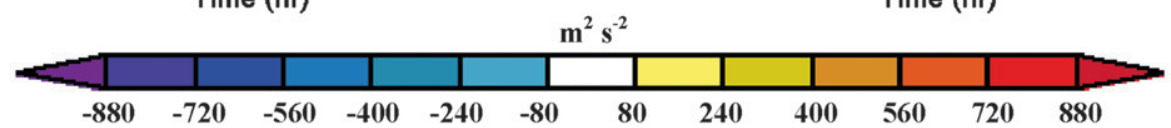

FIG. 17. Vertical Hovmöller diagrams of (left) MMD and (right) EED circulation tendencies $\left(\mathrm{m}^{2} \mathrm{~s}^{-2}\right.$ ) at a radius of $50 \mathrm{~km}$ from domain center for experiments (a) A2, (b) B1, and (c) B2.

2) The system-scale convergence of vorticity contributes less significantly to the development of the systemscale vortex than convective stretching by approximately an order of magnitude.

3) Tilting of horizontal vorticity provides a net negative contribution to the system-scale growth but a positive contribution due to vertical advection negates most of this effect.

4) Moisture significantly affects the symmetry of the system-scale vorticity development. The addition of a surface-to-midlevel moisture anomaly also shortens the gestation period before secondary convection and 
(a)

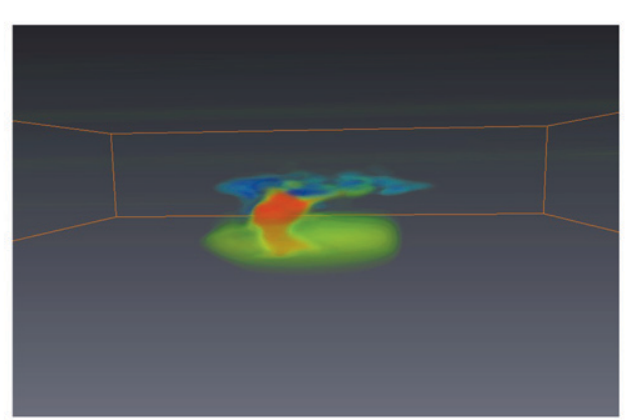

(c)

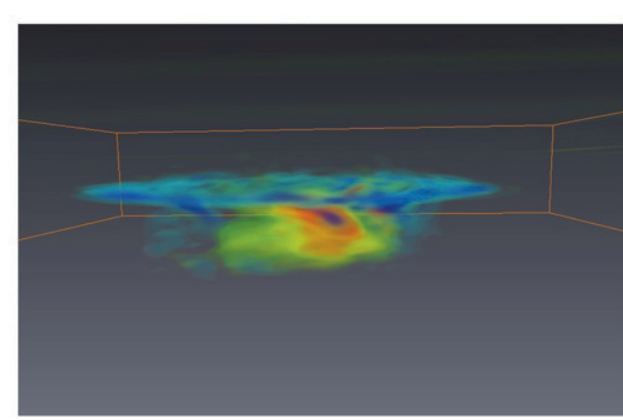

(b)

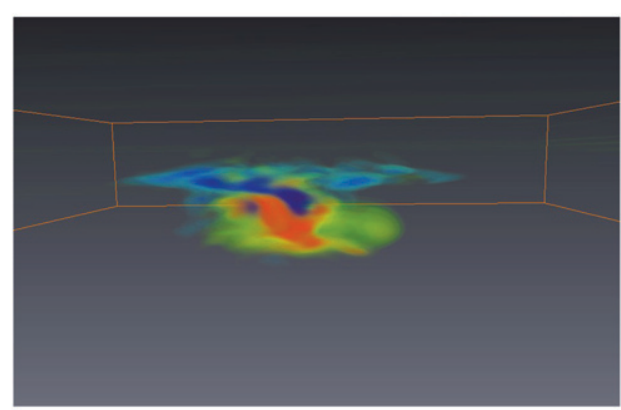

(d)

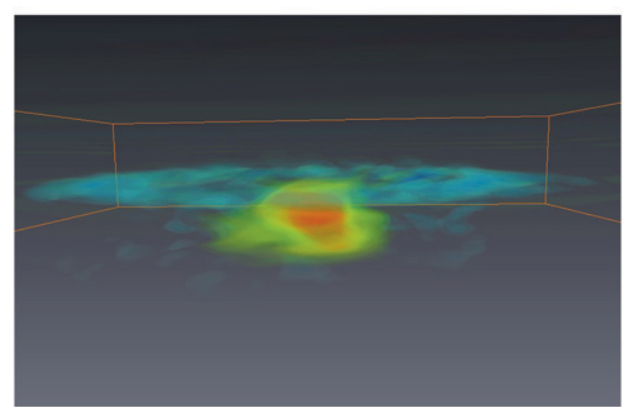

FIG. 18. Three-dimensional representation of system-scale vorticity $\bar{\zeta}$ at $t=$ (a) 24 , (b) 29, (c) 34, and (d) 48 h. Viewed from the south. Light to dark blue indicates negative vorticity, green to red positive.

increases both system- and convective-scale stretching contributions to the mesoscale vortex, consistent with Nolan (2007).

The intentional absence of surface fluxes and friction within the numerical simulations presented herein eliminates any possible intensification due to these processes. Therefore, the net growth of the initial midlevel vortex can be directly attributed to convective rearrangement, generation, or destruction of preexisting vorticity. Convection supplies a source of rotational kinetic energy to the system-scale vortex by tapping into ambient CAPE, at the cost of thermodynamically stabilizing the atmosphere. Otherwise, the initial vortex would only slowly decay because of diffusion. With this in mind and in light of these findings, deep moist convection contributes to the growth of the system-scale vortex in the following manner:

1) Deep convection within an initially cyclonic environment locally stretches ambient vorticity at the surface. With this vorticity seed planted, localized convergence further stretches this cyclonic vorticity at midto upper levels.

2) Multiple discrete diabatic vortex mergers cause an upscale cascade of vorticity through a direct physical increase in their horizontal scale.

3) Once large enough, these vortices begin to interact significantly with the system-scale vortex, enhancing it to form a strong cyclonic core within the larger MCV envelope.

This study confirms the significant role played by VHTs in the formation of the incipient TC vortex. As stated previously, M06 deduced that the primary reason for the system-scale increase in tangential momentum was a net radial influx of system-scale vorticity. They pointed to VHT contributions to the system-scale vorticity as a primary player in this process, referring to the so-called "direct" and "indirect" convective contributions. The direct contribution was to vortically prime the $\mathrm{MCV}$ environment while the indirect effect was to induce a system-scale toroidal circulation that converged both MCV and VHT vorticity. The current study shows through quantification that the direct contribution, or the VHT augmentation of the system-scale vorticity field, is the more significant of these two effects, at least in the initial development within these experiments. However, scale arguments alone cannot rule out the importance of the indirect effect. Naturally, with the inclusion of surface latent heat fluxes, the indirect effect becomes invaluable in sustaining and organizing the larger circulation, especially when working in conjunction with the WISHE mechanism.

A spatial filter can separately evaluate system- and convective-scale features. The spatially filtered terms are both accountants of the large-scale homogeneous flow and projections of the convective-scale heterogeneous 
flow onto the system scale. Thus, the eddy-eddy-divergence (EED) vorticity tendency term does not just capture a summation of localized vorticity stretching, but also the merger of one convective-scale vortex with another. The EED vorticity tendency term captures well the few notable surface vortex merger events detailed in the description of the control simulation and shows that these mergers play a significant role on the growth of the system-scale vortex.

This research has highlighted and quantified the great importance of scale interactions to the intensification of the incipient TC vortex and the criticality of the method used to separate those scales. Although this study has addressed the issue in some depth, further research into this area as applied to TC genesis would be enlightening. Of particular interest is the notion of possible energy surges from the convective scale to the mesoscale, and whether such energy jumps (whether in magnitude or wavenumber) are predictors of TC future intensity. Last, the application of this spatial filtering method on three-dimensional numerical simulations including surface fluxes and friction would be a helpful supplement to the traditional symmetric analysis. Finally, a more comprehensive study on the spectral gap used in the filter design process would provide confirmation of its location for experiments that may have a different spectral gap or be at other stages of TC development.

Acknowledgments. The authors appreciate critical feedback from Dr. Paul Reasor and Dr. Paul Ruscher on prior versions of this research for the first author's M.S. thesis at The Florida State University. The authors are grateful to reviews of this manuscript from Dr. Marc Hidalgo and Dr. Tim Nobis from the Air Force Weather Agency and three anonymous reviewers. The first author would like to especially thank his lovely wife, Mrs. Rebecca Creighton, for her time, emotional support, and grammatical expertise. Most figures in this manuscript were produced using the GrADS software package, provided by the Center for Ocean-LandAtmosphere Studies/Institute of Global Environment and Society. This research was partially supported by NASA Genesis and Rapid Intensification Processes (GRIP) Grant NNX09AC43G.

\section{REFERENCES}

Adem, J., 1956: A series solution for the barotropic vorticity equation and its application to the study of atmospheric vortices. Tellus, 8, 364-372.

Anthes, R. A., 1972: The development of asymmetries in a threedimensional numerical model of a tropical cyclone. Mon. Wea. Rev., 100, 461-476.
Bister, M., and K. A. Emanuel, 1997: The genesis of Hurricane Guillermo: TEXMEX analyses and a modeling study. Mon. Wea. Rev., 125, 2662-2682.

Craig, G. C., and A. Dörnbrack, 2008: Entrainment in cumulus clouds: What resolution is cloud resolving? J. Atmos. Sci., 65, 3978-3988.

Dunkerton, T. J., M. T. Montgomery, and Z. Wang, 2009: Tropical cyclogenesis in a tropical wave critical layer: Easterly waves. Atmos. Chem. Phys., 9, 5587-5646.

Elsberry, R. L., Ed., 1995: Global perspectives on tropical cyclones. WMO Tech. Doc. 693, 289 pp.

Emanuel, K. A., 1986: An air-sea interaction theory for tropical cyclones. Part I: Steady-state maintenance. J. Atmos. Sci., 43, 585-604.

Fiorino, M., and R. L. Elsberry, 1989: Some aspects of vortex structure related to tropical cyclone motion. J. Atmos. Sci., 46, 975-990.

Gray, W. M., 1968: Global view of the origin of tropical cyclones and storms. Mon. Wea. Rev., 96, 669-700.

, 1981: Recent advances in tropical cyclone research from rawinsonde composite analysis. WMO Programme on Research in Tropical Meteorology Rep., 407 pp.

Hendricks, E. A., M. T. Montgomery, and C. A. Davis, 2004: The role of "vortical" hot towers in formation of tropical cyclone Diana (1984). J. Atmos. Sci., 61, 1209-1232.

James, K., 2000: PC Interfacing and Data Acquisition: Techniques for Measurement, Instrumentation and Control. Newnes, $470 \mathrm{pp}$.

Jordan, C. L., 1958: Mean soundings for the West Indies area. J. Meteor., 15, 91-97.

Li, Q., and Y. Wang, 2012: Formation and quasi-periodic behavior of outer spiral rainbands in a numerically simulated tropical cyclone. J. Atmos. Sci., 69, 997-1020.

Lin, Y. L., R. Farley, and H. D. Orville, 1983: Bulk parameterization of the snow field in a cloud model. J. Climate Appl. Meteor., 22, 1065-1092.

Montgomery, M. T., and R. J. Kallenbach, 1997: A theory for vortex Rossby-waves and its application to spiral bands and intensity changes in hurricanes. Quart. J. Roy. Meteor. Soc., 123, 435-465.

— M. E. Nicholls, T. A. Cram, and A. B. Saunders, 2006: A vortical hot tower route to tropical cyclogenesis. J. Atmos. Sci., 63, 355-386.

— S. V. Nguyen, and R. K. Smith, 2009: Do tropical cyclones intensify by WISHE? Quart. J. Roy. Meteor. Soc., 135, 1697-1714.

Moon, Y., and D. S. Nolan, 2010: The dynamic response of the hurricane wind field to spiral rainband heating. J. Atmos. Sci., 67, 1779-1805.

Nolan, D. S., 2007: What is the trigger for tropical cyclogenesis? Aust. Meteor. Mag., 56, 241-266.

- , Y. Moon, and D. P. Stern, 2007: Tropical cyclone intensification from asymmetric convection: Energetics and efficiency. J. Atmos. Sci., 64, 3377-3405.

Petch, J. C., 2006: Sensitivity studies of developing convection in a cloud-resolving model. Quart. J. Roy. Meteor. Soc., 132, 345-358.

Reasor, P. D., M. T. Montgomery, and L. F. Bosart, 2005: Mesoscale observations of the genesis of Hurricane Dolly (1996). J. Atmos. Sci., 62, 3151-3171.

Ritchie, E. A., and G. J. Holland, 1997: Scale interactions during the formation of Typhoon Irving. Mon. Wea. Rev., 125, 13771396.

Rotunno, R., and K. A. Emanuel, 1987: An air-sea interaction theory for tropical cyclones. Part II: Evolutionary study using 
a nonhydrostatic axisymmetric numerical model. J. Atmos. Sci., 44, 542-561.

Schubert, W. H., M. T. Montgomery, R. K. Taft, T. A. Guinn, S. R. Fulton, J. P. Kossin, and J. P. Edwards, 1999: Polygonal eyewalls, asymmetric eye contraction, and potential vorticity mixing in hurricanes. J. Atmos. Sci., 56, 1197-1223.

Simpson, J., E. A. Ritchie, G. J. Holland, J. Halverson, and S. Stewart, 1997: Mesoscale interactions in tropical cyclone genesis. Mon. Wea. Rev., 125, 2643-2661.
Skamarock, W. C., J. B. Klemp, and J. Dudhia, 2001: Prototypes for the WRF (Weather Research and Forecasting) model. Preprints, Ninth Conf. on Mesoscale Processes, Fort Lauderdale, FL, Amer. Meteor. Soc., J11-J15. [Available online at https://ams.confex. com/ams/WAF-NWP-MESO/techprogram/paper_23297.htm.]

Trier, S. B., C. A. Davis, and W. C. Skamarock, 2000: Long-lived mesoconvective vortices and their environment. Part II: Induced thermodynamic destabilization in idealized simulations. Mon. Wea. Rev., 128, 3396-3412. 